\title{
Degrees of Freedom for the MIMO Multi-Way Relay Channel
}

\author{
Ye Tian, Student Member, IEEE, and Aylin Yener, Senior Member, IEEE
}

\begin{abstract}
This paper investigates the degrees of freedom (DoF) of the $L$-cluster, $K$-user MIMO multiway relay channel, where users in each cluster wish to exchange messages within the cluster, and they can only communicate through the relay. A novel DoF upper bound is derived by providing users with carefully designed genie information. Achievable DoF is identified using signal space alignment and multiple-access transmission. For the two-cluster MIMO multiway relay channel with two users in each cluster, the DoF is established for the general case when users and the relay have arbitrary number of antennas, and it is shown that the DoF upper bound can be achieved using signal space alignment or multiple-access transmission, or a combination of both. The result is then generalized to the three user case. For the $L$-cluster $K$-user MIMO multiway relay channel in the symmetric setting, conditions under which the DoF upper bound can be achieved are established. In addition to being shown to be tight in a variety of scenarios of interests of the multiway relay channel, the newly derived upperbound also establishes the optimality of several previously established achievable DoF results for multiuser relay channels that are special cases of the multiway relay channel.
\end{abstract}

Index Terms-MIMO multi-way relay channel, degrees of freedom, signal space alignment, interference alignment.

\section{INTRODUCTION}

$\mathbf{T}$ HE multi-way relay channel [4] is a fundamental building block for relay networks with multicast transmission, and can model several interesting communication scenarios. In cellular networks, a set of mobile users can form a social network by forming clusters and exchange information by communicating via the base station, which serves as the relay in the multi-way relay channel. In ad hoc networks, wireless nodes can be geographically separated, yet they can communicate to a central controller to share information in groups. This model is also relevant to satellite communications, where the satellite serves as the relay and the users have multicast information that needs to be shared with the help of the satellite [4].

Manuscript received July 30, 2013; revised February 6, 2014; accepted February 7, 2014. Date of publication March 4, 2014; date of current version April 17, 2014. This work was supported by NSF under Grants 0964364 and 0964362. This paper was presented at the 2012 IEEE International Conference on Communications in China [1], the 2013 IEEE International Conference on Communications [2], and the 2013 IEEE International Symposium on Information Theory [3].

Y. Tian was with the Department of Electrical Engineering, Pennsylvania State University, University Park, PA 16802 USA. He is now with Broadcom Corporation, Sunnyvale, CA 94085 USA (e-mail: yetian@broadcom.com).

A. Yener is with the Department of Electrical Engineering, Pennsylvania State University, University Park, PA 16802 USA (e-mail: yener@ee.psu.edu). Communicated by A. Lozano, Associate Editor for Communications.

Color versions of one or more of the figures in this paper are available online at http://ieeexplore.ieee.org.

Digital Object Identifier 10.1109/TIT.2014.2309646
The simplest special case of the multi-way relay channel is the two-way relay channel, which consists of two users that wish to exchange information with the help of a relay. The capacity of the two-way relay channel has been studied extensively, see for example [5]-[9] and the references therein. Even for this simplest set up, only constant gap capacity results is known [7], achieved by physical layer network coding, or functional decode-and-forward (FDF).

In its general form, the multi-way relay channel, contains an arbitrary number of clusters containing arbitrary number of users that want to exchange information. The relay needs to handle interference that results from simultaneous transmissions of different clusters, and the users need to recover the intended messages in the presence of interfering signals containing messages for other users. One might expect the strategies designed for the two-way relay channel to be helpful, but more sophisticated strategies are needed to handle the co-existence of messages intended for different users.

The exact capacity characterization for the multi-way relay channel has been considered in references [4] and [10]-[14]. Specifically, reference [4] has proposed the general multi-way relay channel model, and characterized the upperbounds on the capacity region and established achievable rates based on decode-and-forward (DF), compress-and-forward (CF), amplify-and-forward (AF), and using nested lattice codes. Reference [10] has considered the special case when there is one cluster of users, and each user wishes to exchange information with the rest of the users. The capacity region is characterized for a finite field channel. It is shown that, for this case, functional-decode-forward (FDF) combined with rate splitting and joint source-channel coding achieves capacity. For the Gaussian multi-way relay channel with one cluster, capacity result is obtained for some special cases when the channel is symmetric using FDF [11]. For the asymmetric multi-way relay channel with a single cluster, also known as the Y channel, references [12] and [13] have obtained a constant gap capacity result for all channel coefficient values for the three-user case. Reference [14] has studied the multi-way relay channel with two clusters and each cluster has two users with a single antenna, and established a constant gap capacity result using a combination of lattice codes and Gaussian codes. For the multi-cluster set up with two users in each cluster, i.e., the multi-pair two-way relay channel, reference [15] has studied the detection and interference management strategies, and reference [9] has studied power allocation with orthogonal channels. For the general multi-way relay channel, the exact 
capacity remains unknown due to the complexity of channel, in turn making it difficult to obtain design insights for the general setting.

While the capacity characterizes the exact region of rate pairs that allow reliable communication in a communication system, the degrees of freedom (DoF), characterizes the approximate capacity of a communication system: it studies how the reliable communication rate scales with power as power goes to infinity, or the prelog factor of the capacity. Studying DoF can provide valuable insights about the optimal signal interaction in time/frequency/space dimensions, leading to design of transmission schemes to achieve higher rates. ${ }^{1}$ One should note that the design insights relate to local interactions of nodes and care must be exercised from drawing conclusions on network-wide performance when such a small network is embedded in a general topology wireless network. Additionally, one should note that care must be exercised in applying and implementing DoF optimal strategies in finite SNR regime.

Interference alignment establishes the optimal $\mathrm{DoF}$ for various multi-user wireless network models [16]-[19]. The essence of interference alignment lies in keeping the interference signals in the smallest number of time/frequency/space dimensions, and enabling the maximum number of independent data streams to be transmitted. A similar concept, signal space alignment, which is a special form of FDF, is proposed in reference [20] for the $Y$ channel. In this reference, the authors have shown that, by aligning the signals from the users that want to exchange information at the same dimension, network coding can be utilized to maximize the utilization of the spatial dimension available at the relay to achieve the optimal DoF. In essence, the goal of signal space alignment is to align the useful signals together to maximize the utilization of signal dimension, whereas the goal of interference alignment is to align the harmful signals together to minimize the effect of interference. In reference [21], the signal space alignment idea is extended to the $K$-user Y channel, which has $K$ users in a single cluster that want to exchange information, and the achievable DoF is established. For the MIMO multi-pair two-way relay channel, reference [22] has studied the requirement for the number of antennas at the users to allow them exchange information with the help of the relay without interfering each other in the symmetric setting. The DoF of this channel is further studied in reference [23]. Signal space alignment is further utilized in reference [24], which has considered a different variation of the MIMO multiway relay channel where a base station wants to exchange information with $K$ users with the help of a relay. The DoF of this model is established under some specific relations between the number of antennas at the relay and at the users.

For the DoF characterization of MIMO multi-way relay channels, the known DoF upperbound obtained to date is a cut-set bound, which can provide a tight upperbound for the two-way relay channel, three-user $Y$ channel and

\footnotetext{
${ }^{1}$ For low SNR, optimal strategies may differ from those offered by the DoF analysis.
}

two-cluster multiway relay channel with two users in each cluster, but can be arbitrarily loose for other instances of the model.

In this work, we derive a new DoF upper bound for the $L$-cluster $K$-user MIMO multi-way relay channel using a genie-aided approach, such that the user with enhanced signal and a carefully designed set of genie information can decode a subset of messages from the other users. We show that the DoF for the MIMO multi-way relay channel is always upper bounded by $2 N$ with $N$ being the number of antennas at the relay. This DoF upper bound, combined with the cut-set bound, provides us a comprehensive set of DoF upper bounds for the general MIMO multi-way relay channel. This allows us to show that the DoF upperbound is tight for some achievable DoF results in previous works corresponding to special cases of the multi-way relay channel.

Next, we investigate the achievable DoF for several scenarios of the MIMO multi-way relay channel. We utilize the idea of signal space alignment in [20]-[22], where the users utilize the signal space of the relay in common, and the relay can decode a function of the transmitted signals from a pair of users and multiple-access transmission, where the users do not share the signal space of the relay, and the relay simply decodes the transmitted signals as in the multiple access channel to establish the achievability results.

For clarity, we first consider the case with two clusters and each cluster has two users, and the users and the relay can have arbitrary number of antennas. We show that for some cases, signal space alignment achieves the optimal DoF. For the remaining cases, the DoF upper bound can be achieved using multiple-access transmission or a combination of multipleaccess transmission and signal space alignment. Additionally, for some cases, using only a subset of antennas at the relay is sufficient to achieve the optimal DoF. We next generalize the results to the case with two clusters and each cluster has three users, and obtain the optimal DoF for several scenarios of interests. We then consider the $L$-cluster $K$-user MIMO multi-way relay channel in the symmetric setting, where all users have the same number of antennas. Conditions between the number of antennas at the relay and the users are established when the DoF upper bound can be achieved. The DoF result implies that the DoF for the MIMO multi-way relay channel is always limited by the spatial dimension available at the relay, and increasing the number of users and clusters cannot achieve DoF gain when the number of antennas at the relay is limited. Furthermore, since using signal space alignment to share the signal space of the relay between two users can provide 1 bit for 2 bits gain, the DoF upper bound $2 \mathrm{~N}$ provides the insight that we cannot obtain any further DoF gain by letting three or more users to share the same spatial dimension of the relay.

The remainder of the paper is organized as follows. Section II describes the channel model. Section III derives the new DoF upper bound for the general MIMO multi-way relay channel. Section IV investigates the DoF for the twocluster MIMO multi-way relay channel. Section $\mathrm{V}$ investigates the DoF for the general MIMO multi-way relay channel. Section VI concludes the paper. 


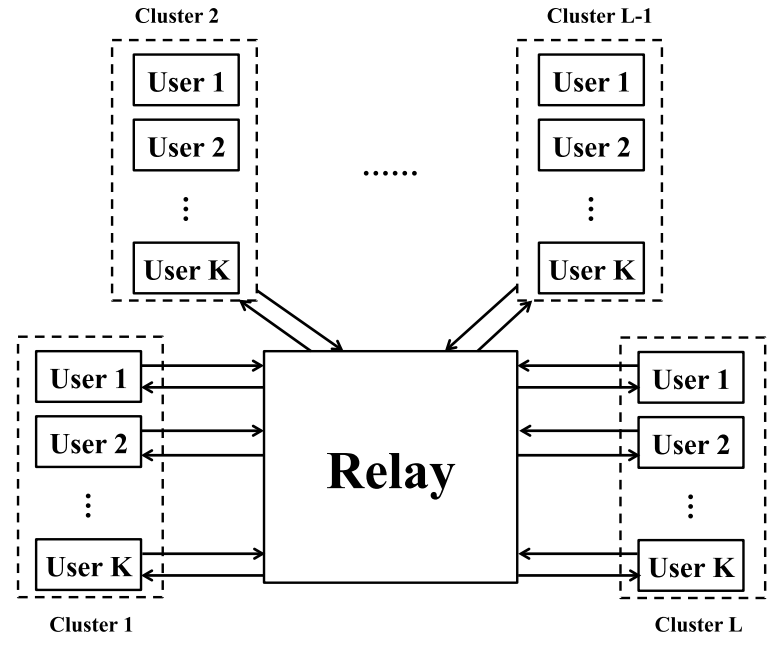

Fig. 1. K-user L-cluster MIMO multi-way relay channel.

\section{Channel Model}

The $L$-cluster $K$-user MIMO multi-way relay channel is shown in Fig. 1. User $k(k=1,2, \ldots, K)$ in cluster $l$ $(l=1,2, \ldots, L)$ is assumed to have $M_{k}^{l}$ antennas, and the relay is assumed to have $N$ antennas. Without loss of generality, we assume that $M_{1}^{l} \geq M_{2}^{l} \geq \cdots \geq M_{K}^{l}$.

In cluster $l$, user $k$ has a message $W_{i k}^{l}(i=1,2, \ldots, K$, $i \neq k$ ), for all the other users in cluster $l$. We denote $\mathcal{W}_{k}^{l}$ as the message set originated from user $k$ in cluster $l$ for all the other users in the same cluster, i.e.,

$$
\mathcal{W}_{k}^{l}=\left\{W_{1 k}^{l}, W_{2 k}^{l}, \ldots, W_{k-1, k}^{l}, W_{k+1, k}^{l}, \ldots, W_{K k}^{l}\right\} .
$$

It is assumed that the users can communicate only through the relay and no direct links exist between any pairs of users [4]. All the nodes in the network are assumed to be full duplex. The transmitted signal from user $k$ in cluster $l$ for channel use $t$ is denoted as $\mathbf{X}_{k, l}(t) \in \mathbb{C}^{M_{k}^{l}}$. The received signal at the relay for channel use $t$ is denoted as $\mathbf{Y}_{R}(t) \in \mathbb{C}^{N}$. The received signal at user $k$ in cluster $l$ for channel use $t$ is defined as $\mathbf{Y}_{k, l}(t) \in \mathbb{C}^{M_{k}^{l}}$. The channel matrix from user $k$ in cluster $l$ to the relay is denoted as $\mathbf{H}_{R(k, l)}(t) \in \mathbb{C}^{N \times M_{k}^{l}}$. The channel matrix from the relay to user $k$ in cluster $l$ is denoted as $\mathbf{H}_{(k, l) R}(t) \in \mathbb{C}^{M_{k}^{l} \times N}$. It is assumed that the entries of the channel matrices are drawn independently from a continuous distribution, which guarantees that the channel matrices are full rank almost surely.

The encoding function at user $k$ in cluster $l$ is defined as

$$
\mathbf{X}_{k, l}(t)=f_{k, l}\left(\mathcal{W}_{k}^{l}, \mathbf{Y}_{k, l}^{t-1}\right),
$$

where $\mathbf{Y}_{k, l}^{t-1}=\left[\mathbf{Y}_{k, l}(1), \ldots, \mathbf{Y}_{k, l}(t-1)\right]$.

The received signal at the relay is

$$
\mathbf{Y}_{R}(t)=\sum_{l=1}^{L} \sum_{k=1}^{K} \mathbf{H}_{R(k, l)}(t) \mathbf{X}_{k, l}(t)+\mathbf{Z}_{R}(t) .
$$

For channel use $t$, the transmitted signal $\mathbf{X}_{R}(t) \in \mathbb{C}^{N}$ from the relay is a function of its received signals from channel use 1 to $t-1$, i.e.,

$$
\mathbf{X}_{R}(t)=f_{R}\left(\mathbf{Y}_{R}^{t-1}\right)
$$

The received signal at user $k$ in cluster $l$ for channel use $t$ is

$$
\mathbf{Y}_{k, l}(t)=\mathbf{H}_{(k, l) R}(t) \mathbf{X}_{R}(t)+\mathbf{Z}_{k, l}(t) .
$$

In the above expressions, $\mathbf{Z}_{k, l}(t) \in \mathbb{C}^{M_{k}^{l}}, \mathbf{Z}_{R}(t) \in \mathbb{C}^{N}$ are additive white Gaussian noise vectors with zero mean and independent components. The transmitted signals from the users and the relay satisfy the following power constraints:

$$
\begin{aligned}
E\left[\operatorname{tr}\left(\mathbf{X}_{k, l}(t) \mathbf{X}_{k, l}(t)^{\dagger}\right)\right] & \leq P, \\
E\left[\operatorname{tr}\left(\mathbf{X}_{R}(t) \mathbf{X}_{R}(t)^{\dagger}\right)\right] & \leq P .
\end{aligned}
$$

Based on the received signals and the message set $\mathcal{W}_{k}^{l}$, user $k$ in cluster $l$ needs to decode all the messages intended for it, which is denoted as

$$
\hat{\mathcal{W}}_{k}^{l}=\left\{\hat{W}_{k, 1}^{l}, \hat{W}_{k, 2}^{l}, \ldots, \hat{W}_{k, k-1}^{l}, \hat{W}_{k, k+1}^{l}, \ldots, \hat{W}_{k, K}^{l}\right\} .
$$

We also have

$$
\hat{\mathcal{W}}_{k}^{l}=g_{k, l}\left(\mathbf{Y}_{k, l}^{n}, \mathcal{W}_{k}^{l}\right)
$$

where $g_{k, l}$ is the decoding function for user $k$ in cluster $l$.

We assume the rate of message $W_{i k}^{l}$ is $R_{i k}^{l}(P)$ under power constraint $P$. A rate tuple $\left\{R_{i k}^{l}(P)\right\}$ with $l=1, \ldots, L$, $k=1, \ldots, K$ and $i=1, \ldots, K, i \neq k$ is achievable if the error probability

$$
P_{e}^{n}=\operatorname{Pr}\left(\bigcup_{l, k, i} \hat{W}_{i, k}^{l} \neq W_{i, k}^{l}\right) \rightarrow 0
$$

as $n \rightarrow \infty$.

We define $\mathcal{C}(P)$ as the set of all achievable rate tuples $\left\{R_{i k}^{l}(P)\right\}$, under power constraint $P$. The degrees of freedom is defined as

$$
D o F=\lim _{P \rightarrow \infty} \frac{R_{\sum}(P)}{\log (P)},
$$

where

$$
R_{\Sigma}(P)=\sup _{\left\{R_{i k}^{l}(P)\right\} \in \mathcal{C}(P)} \sum_{l=1}^{L} \sum_{k=1}^{K} \sum_{\substack{i=1 \\ i \neq k}}^{K} R_{i k}^{l}(P)
$$

is the sum capacity under power constraint $P$.

\section{DOF UPPERBOUND FOR GENERAL MIMO Multi-Way Relay ChanNel}

Theorem 1: For the general $L$-cluster $K$-user MIMO multi-way relay channel, the DoF upperbound is

$$
D o F \leq \min \left\{\sum_{l=1}^{L}\left(\sum_{i=2}^{K} M_{i}^{l}+\min \left\{M_{1}^{l}, \sum_{k=2}^{K} M_{k}^{l}\right\}\right), 2 N\right\} \text {. }
$$

Proof: The first term of the upperbound can be derived using a cut set bound as follows. Note that by assumption we have $M_{1}^{l} \geq M_{2}^{l} \geq \cdots \geq M_{K}^{l}$. For the messages in cluster $l$, we give the users in cluster $l$ all the messages from all the other clusters. We also provide the relay all the messages from all the clusters except cluster $l$, and provide all the other clusters all the messages from all clusters. This operation does not reduce the rate of the messages in cluster $l$. Now the channel 
is effectively a MIMO multi-way relay channel with a single cluster. For the messages intended for user $i$, we can combine all the other users except for user $i$, which yields a two-way relay channel with user $i$ as a node, and all the other users as a node. We can then bound the DoF for the messages in cluster $l$ in the following fashion:

$$
\begin{aligned}
\sum_{k=2}^{K} d_{1 k}^{l} & \leq \min \left\{M_{1}^{l}, N, \sum_{k=2}^{K} M_{k}^{l}\right\} \\
\sum_{\substack{k=1 \\
k \neq i, i \neq 1}}^{K} d_{i k}^{l} & \leq \min \left\{M_{i}^{l}, N\right\} .
\end{aligned}
$$

This yields the desired DoF upperbound for the first term in (13).

To prove $D o F \leq 2 N$, we consider user 1 in each cluster. For user 1 in cluster $l$, we provide user 1 with all the received signals $\mathbf{Y}_{k, l}^{n}$ from user $k=2,3, \ldots, K$ in cluster $l$ as genie information. ${ }^{2}$ By assumption, user $k$ in cluster $l$ can decode messages $\left\{W_{k i}^{l}\right\}$ for $i=\{1, \ldots, K\} \backslash\{k\}$ given the received signal $\mathbf{Y}_{k, l}^{n}$ and the side information $\mathcal{W}_{k}^{l}$. We now use the following steps to derive the DoF upper bound.

- Step 1:

- User 1 in cluster $l$ has side information $\mathcal{W}_{j 1}^{l}$, $j=\{2, \ldots, K\}$, which are messages that originate from it.

- User 1 in cluster $l$ can decode messages $\left\{W_{1 i}^{l}\right\}$ for $i=\{2, \ldots, K\}$.

- Step 2:

- Let a genie provide user 1 in cluster $l$ with messages $\left\{W_{i 2}\right\}, i=\{3, \ldots, K\}$.

- User 1 now has the messages $\left\{W_{i 2}\right\}, i=$ $\{1,3, \ldots, K\}$, which is exactly all the side information available at user 2 in cluster $l$.

- With the genie information $\mathbf{Y}_{2, l}^{n}$, user 1 in cluster $l$ can decode all the messages intended for user 2 in cluster $l$, i.e., the messages $\left\{W_{2 i}^{l}\right\}$ for $i=$ $\{3, \ldots, K\}$

- Step 3:

- Let a genie provide user 1 in cluster $l$ with messages $\left\{W_{i 3}\right\}, i=\{4, \ldots, K\}$.

- User 1 now has the messages $\left\{W_{i 3}\right\}, i=$ $\{1,2,4, \ldots, K\}$, which is exactly all the side information available at user 3 in cluster $l$.

- With the genie information $\mathbf{Y}_{3, l}^{n}$, user 1 in cluster $l$ can decode the messages $\left\{W_{3 i}^{l}\right\}$ for $i=\{4, \ldots, K\}$.

- Proceed in the same fashion for the next steps, i.e., for Step $k$ :

- Let a genie provide user 1 in cluster $l$ with messages $\left\{W_{i k}\right\}, i=\{k+1, \ldots, K\}$.

- User 1 now has the messages $\left\{W_{i k}\right\}, i=\{1, \ldots, K\} /$ $\{k\}$, which is exactly all the side information available at user $k$ in cluster $l$.

\footnotetext{
${ }^{2}$ Alternatively, we can use a channel enhancement argument to prove the DoF upperbound, as shown in [3].
}

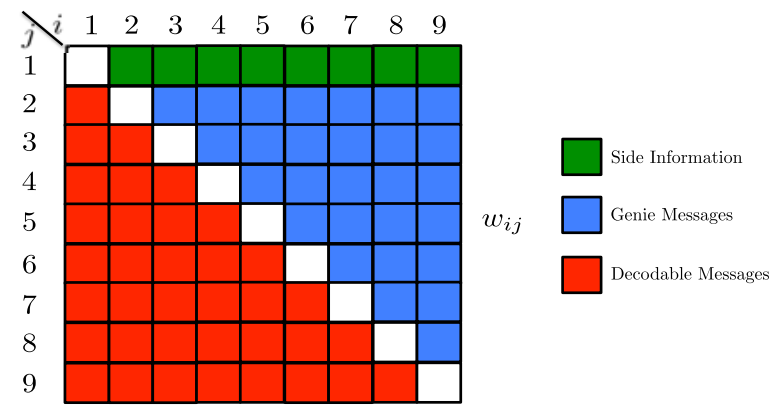

Fig. 2. Illustration for side information, genie information and decodable messages for the DoF upperbound at user 1.

- With the genie information $\mathbf{Y}_{k, l}^{n}$, user 1 in cluster $l$ can decode the messages $\left\{W_{k i}^{l}\right\}$ for $i=\{k+$ $1, \ldots, K\}$.

- Step $K-1$ :

- Let a genie provide user 1 in cluster $l$ with message $W_{K, K-1}^{l}$.

- User 1 now has the messages $\left\{W_{i, K-1}\right\}, i=$ $\{1, \ldots, K-2, K\}$, which is exactly all the side information available at user $K-1$ in cluster $l$.

- User 1 in cluster $l$ can decode the message $W_{K-1, K}$.

Based on the above arguments, user 1 in cluster $l$ can decode the messages $\left\{W_{k i}^{l}\right\}$ for $k=1, \ldots, K-1, i=k+1, \ldots, K$. This upperbounding process is illustrated in Fig. 2 for $K=9$. We can see that half of the messages in cluster $l$ can be decoded at user 1 in cluster $l$ based on the received signals $\mathbf{Y}_{k, l}^{n}, k=1, \ldots, K$, and the other half of the messages in the cluster as side information, which include the messages $\mathcal{W}_{1}^{l}$ and the genie information $\left\{W_{i k}^{l}\right\}$ for $k=2, \ldots, K-1$, $i=k+1, \ldots, K$.

Define $\mathcal{W}_{d}^{l}$ as the set of messages $\left\{W_{k i}^{l}\right\}$ for $k=1, \ldots, K-1$, $i=\{k+1, \ldots, K\}$ for cluster $l$, which are messages that can be decoded by user 1 in cluster $l$, and $\mathcal{W}^{l}$ as all the messages from cluster $l$ and $\mathcal{W}_{d}^{l c}$ as the set of messages $\mathcal{W}^{l} / \mathcal{W}_{d}^{l}$. Denote the set of received signals $\mathbf{Y}_{k, l}, k=1, \ldots, K$ in cluster $l$ by $\mathcal{Y}_{l}$. We can then bound the rate of the decodable messages as follows:

$$
\begin{aligned}
n \sum_{l=1}^{L} & \sum_{k=1}^{K-1} \sum_{i=k+1}^{K} R_{k i}^{l} \\
= & H\left(\mathcal{W}_{d}^{1}, \ldots, \mathcal{W}_{d}^{L} \mid \mathcal{W}_{d}^{1 c}, \ldots, \mathcal{W}_{d}^{L c}\right) \\
= & I\left(\mathcal{W}_{d}^{1}, \ldots, \mathcal{W}_{d}^{L} ; \mathcal{Y}_{1}^{n}, \ldots, \mathcal{Y}_{L}^{n} \mid \mathcal{W}_{d}^{1 c}, \ldots, \mathcal{W}_{d}^{L c}\right)+n \epsilon_{n} \\
\leq & H\left(\mathcal{Y}_{1}^{n}, \ldots, \mathcal{Y}_{L}^{n}\right) \\
& -H\left(\mathcal{Y}_{1}^{n}, \ldots, \mathcal{Y}_{L}^{n} \mid \mathcal{W}^{1}, \ldots, \mathcal{W}^{L}, X_{R}^{n}\right)+n \epsilon_{n} \\
= & H\left(\mathcal{Y}_{1}^{n}, \ldots, \mathcal{Y}_{L}^{n}\right) \\
& -H\left(\mathcal{Y}_{1}^{n}, \ldots, \mathcal{Y}_{L}^{n} \mid X_{R}^{n}\right)+n \epsilon_{n} \\
= & I\left(X_{R}^{n} ; \mathcal{Y}_{1}^{n}, \ldots, \mathcal{Y}_{L}^{n}\right)
\end{aligned}
$$

where equation (20) follows since the received signal at the users only depends on the transmitted signal from the relay. 


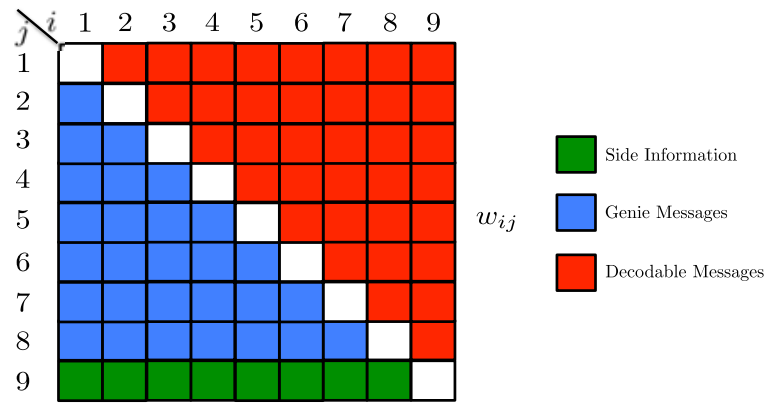

Fig. 3. Illustration for side information, genie information and decodable messages for the DoF upperbound at user $K$.

From equation (21), we can see that

$$
\lim _{S N R \rightarrow \infty} \frac{\sum_{l=1}^{L} \sum_{k=1}^{K-1} \sum_{i=k+1}^{K} R_{k i}^{l}}{\log S N R} \leq N .
$$

We now have an upper bound for half of the messages from all users. We can bound the DoF for the rest of the messages by enhancing the received signal of user $K$ and provide genie information to user $K$ in a similar fashion, as illustrated in Fig. 3, which yields

$$
\lim _{S N R \rightarrow \infty} \frac{\sum_{l=1}^{L} \sum_{k=2}^{K} \sum_{i=1}^{k-1} R_{k i}^{l}}{\log S N R} \leq N .
$$

Given (22) and (23), we have

$$
D o F \leq 2 N \text {. }
$$

\section{A. Optimality of Achievable DoF for Special Cases in Previous Work}

We can now evaluate the optimality of the achievable DoF for some special cases of the MIMO multi-way relay channel provided in previous work using the newly derived DoF upperbound.

- MIMO K-user Y channel [21]: This is the MIMO multiway relay channel with one cluster that contains $K$ users. User $i$ has $M_{i}$ antennas and the relay has $N$ antennas. It was shown in [21] that each user can send $K-1$ independent data streams with DoF $d$ for each stream if

$$
\begin{aligned}
M_{i} & \geq d(K-1), N \geq \frac{d K(K-1)}{2} \\
N & <\min \left\{M_{i}+M_{j}-d \mid \forall i \neq j\right\} .
\end{aligned}
$$

For this case, our DoF upperbound specializes to

$$
D o F \leq \min \left\{\sum_{i=1}^{K} M_{i}, 2 N\right\}
$$

If we have $M_{i} \geq d(K-1)$ and fix $N=\frac{d K(K-1)}{2}$, the DoF upper bound becomes

$$
D o F \leq d K(K-1) .
$$

If we further have $M_{i}>\frac{K^{2}-K+2}{4}$, the condition $N<$ $\min \left\{M_{i}+M_{j}-d \mid \forall i \neq j\right\}$ is also satisfied, and the DoF upper bound implies that the achievable DoF in [21], $d K(K-1)$, is indeed the optimal DoF.
- MIMO K-pair two-way relay channel [22]: This corresponds to the MIMO multi-way relay channel with $K$ clusters each with two users. Each user has $M$ antennas and wants to transmit $d$ data streams with DoF 1 . The relay has $K d$ antennas. To guarantee interference-free transmission, we need

$$
K \leq \frac{2 M}{d}-1
$$

and the achievable DoF is $2 K d$.

For this case, the DoF upper bound becomes

$$
D o F \leq \min \{2 K M, 2 K d\} .
$$

When $K \leq \frac{2 M}{d}-1$, we have $2 K d \leq 2 K M$ for $K \geq 2$. The achievable DoF $2 K d$ given in reference [22] is indeed the optimal DoF.

We now have seen that the newly derived upper bound is useful for proving tight results for some special cases of the MIMO multi-way relay channel. In the next sections, we utilize the upper bound to investigate the DoF of the more general MIMO multi-way relay channel, and provide our DoF findings.

\section{Two-Cluster Mimo Multi-Way Relay Channel}

With the newly derived DoF upper bound at hand, we now investigate the achievable DoF for the general MIMO multi-way relay channel. We first focus on the two-cluster MIMO multi-way relay channel. For the two-cluster case, the only known result is the constant gap capacity result for the SISO case [14] and the DoF for the two-user symmetric case [22], i.e., users have the same number of antennas. Both results are obtained using signal space alignment, or using techniques that are in essence similar to signal space alignment such as using nested lattice codes [25]. When the users have arbitrary number of antennas, the optimal DoF has been unknown to date.

We first present the following lemma which characterizes the dimension of shared signal space at the relay between two users with arbitrary number of antennas.

Lemma 1: For matrices $\mathbf{H}_{1} \in \mathbb{C}^{p \times q_{1}}$ and $\mathbf{H}_{2} \in \mathbb{C}^{p \times q_{2}}$, which have full rank almost surely, the shared dimension of their column space can be specified as follows. Note that without loss of generality we assume $q_{1} \geq q_{2}$.

Condition 1: If $p \geq q_{1} \geq q_{2}$ and $q_{1}+q_{2}>p$, then there exist $q_{1}+q_{2}-p$ non-zero linearly independent vectors $\mathbf{v}_{i}$ almost surely such that we can find another two sets of linearly independent vectors $\mathbf{u}_{i}$ and $\mathbf{w}_{i}, i=1, \ldots, q_{1}+q_{2}-p$ such that

$$
\mathbf{v}_{i}=\mathbf{H}_{1} \mathbf{u}_{i}=\mathbf{H}_{2} \mathbf{w}_{i} .
$$

Condition 2: If $q_{1} \geq p \geq q_{2}$, then there exist $q_{2}$ linearly independent vectors $\mathbf{v}_{i}$ almost surely such that we can find another two sets of linearly independent vectors $\mathbf{u}_{i}$ and $\mathbf{w}_{i}$, $i=1, \ldots, q_{2}$ such that

$$
\mathbf{v}_{i}=\mathbf{H}_{1} \mathbf{u}_{i}=\mathbf{H}_{2} \mathbf{w}_{i}
$$

Proof: The proof is provided in Appendix A. 
Remark 1: The result in Condition 1 is the same as the result in [20]. For this case, the dimension of the shared signal space decreases as the number of antennas at the relay increases.

Remark 2: Condition 2 implies that, when there is asymmetry between the number of antennas at the users, the dimension of shared signal space between two users cannot exceed the dimension of the user with the smallest number of antennas.

\section{A. Two Users in Each Cluster: Optimal DoF}

Theorem 2: Consider the two-cluster MIMO multi-way relay channel with two users in each cluster as described in Section II. Without loss of generality, assume $M_{2}^{1} \geq M_{2}^{2}$. The optimal DoF and the DoF achieving strategies are described in the tables below:

(1) $D o F^{*}=2 N$ See Table I.

(2) $D o F^{*}=2\left(M_{2}^{1}+M_{2}^{2}\right)$ See Table II.

In the tables above, "Two-way RC with FDF" indicates that only one cluster is active during transmission, which reduces the channel to a two-way relay channel, and functional decodeand-forward (FDF) achieves the optimal DoF. "SSA at both clusters" implies that both clusters use signal space alignment [20]-[22] to share the signal spaces at the relay. "MAC+BC" denotes the scheme where users simply send signals to the relay as a multiple-access channel, and the relay decodes and broadcasts the intended messages back to the users.

Proof: The achievability of the above DoF and a more general achievable DoF result are provided in Proposition 1 in Appendix B. The optimality follows from the DoF upperbound we derived in Theorem 1.

Studying the general multi-way relay channel where users have arbitrary number of antennas can reveal some interesting cases when the optimal DoF can be established, which are not revealed by the symmetric case. For example, case II.B along with the scheme used in Appendix A and B show that the asymmetry in the number of antennas at the users can be utilized to facilitate signal space alignment: when one user has more antennas than the relay, the other user can send its signals along any direction. The user with more antennas can adjust its signal direction to align with the other user at the relay, and no coordination between the two users is needed. The achievable schemes for the optimal DoF for cases IV.B.1, IV.C.1, IV.D.2 and IV.D.3 show that multiple-access transmission scheme can be combined with signal space alignment, and can be useful to achieve the optimal DoF. For all the conditions in case IV, using a subset of the antennas at the relay is sufficient to achieve the optimal DoF. Using all the antennas may be able to improve the achievable rates, but cannot provide any further DoF. A numerical example is provided in Fig. 4 to illustrate this. The number of antennas for this example are $N=3, M_{1}^{1}=4, M_{2}^{1}=1, M_{1}^{2}=3, M_{2}^{2}=1$, corresponding to case IV.A. The achievable rate is calculated using the scheme in Appendix A and B. We can see that using 2 antennas at the relay, the same DoF can be achieved as using 3 antennas. Using 3 antennas can provide higher achievable rates, but not a higher slope. In the plots, we also provided the achievable rate using $\mathrm{MAC}+\mathrm{BC}$ with zero-forcing precoder/decoder using all

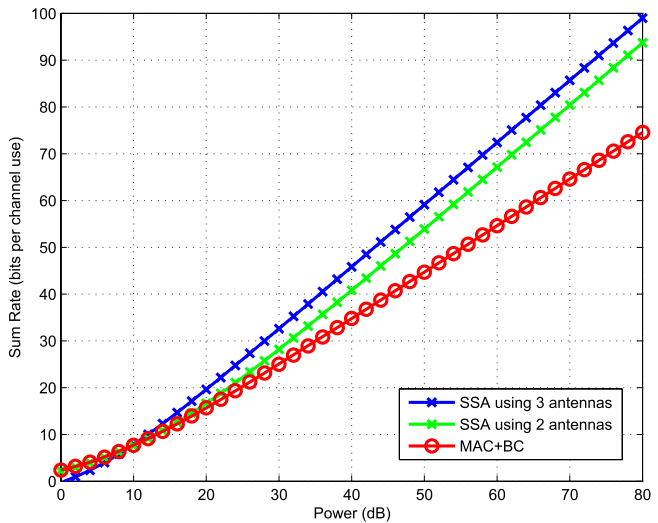

Fig. 4. Achievable rates using signal space alignment.

available antennas, and we can see that the achievable rate is strictly less than using SSA even with 2 antennas at the relay at moderate SNR.

Remark 3: Note that for cases IV.B.2, IV.C.2 and IV.D, to guarantee that the number of shared signal dimensions at the relay is maximized for our signal space alignment scheme, relay must use a subset of the antennas. We note that there may exist more sophisticated schemes that can also achieve the same optimal DoF using all the antennas at the relay, for example, the schemes developed in [26]. Meanwhile, in finite SNR scenarios, using all the antennas at the relay can be beneficial, as this can provide power gain using maximum ratio combining type of schemes, especially for low SNR cases.

\section{B. Two Users in Each Cluster: Symmetric Case}

We now consider the two-cluster MIMO multi-way relay channel with two users in each cluster with $M_{1}^{1}=M_{1}^{2}=M_{1}$ and $M_{2}^{1}=M_{2}^{2}=M_{2}$. The optimal DoF for this special case is summarized as follows:

Corollary 1: For the two-cluster MIMO multi-way relay channel with two users in each cluster with $M_{1}^{1}=M_{1}^{2}=M_{1}$ and $M_{2}^{1}=M_{2}^{2}=M_{2}$ (without loss of generality assume $M_{1} \geq M_{2}$ ), the optimal DoF is:

When $N \leq 2 M_{2}$,

- $N \leq M_{2}, D_{o} F^{*}=2 N$

- $M_{2}<N \leq M_{1}, D o F^{*}=2 N$.

- $M_{2} \leq M_{1}<N \leq \frac{2}{3}\left(M_{1}+M_{2}\right)$, DoF $F^{*}=2 N$.

When $N>2 M_{2}$,

- $N \geq 4 M_{2}, D o F^{*}=4 M_{2}$.

- $N<4 M_{2}, N \leq M_{1}, D o F^{*}=4 M_{2}$.

- $N>M_{1} \geq 2 M_{2}, D o F^{*}=4 M_{2}$.

Proof: This corollary follows as a special case from Proposition 1, and the upperbound in Theorem 1.

Fig. 5 illustrates the regimes for which we can establish the optimal DoF for the symmetric case with $N=16$. In the figure, Two-way $R C$ denotes the region where the DoF can be achieved by only allowing one cluster to exchange data with the relay, which reduces the channel to a two-way relay channel. $M A C+B C$ denotes the region that the users use multipleaccess transmission and the relay decodes and broadcasts the messages to the intended users. SSA represents signal space 
TABLE I

Two-User CASE: $D o F^{*}=2 N$

\begin{tabular}{|l|l||l|}
\hline \multicolumn{2}{|c|}{ Conditions when optimal $D o F^{*}$ can be achieved } & Optimal Strategy \\
\hline \multicolumn{1}{|c||}{ I. $N \leq M_{2}^{1}$} & Two-way RC with FDF \\
\hline & A. $N \leq M_{1}^{1}$ and $N \leq M_{1}^{2}$ & SSA for both clusters \\
\cline { 2 - 3 } II. $M_{2}^{1}+M_{2}^{2} \geq N>M_{2}^{1} \geq M_{2}^{2}$ & $\begin{array}{l}\text { B. } M_{1}^{1}<N \leq M_{1}^{2} \text { and } \\
M_{1}^{1}+M_{2}^{1}+M_{2}^{2} \geq 2 N\end{array}$ & SSA for both clusters \\
\cline { 2 - 3 } & $\begin{array}{l}\text { C. } M_{1}^{2}<N \leq M_{1}^{1} \text { and } \\
\text { and } M_{2}^{1}+M_{1}^{2}+M_{2}^{2} \geq 2 N\end{array}$ & SSA for both clusters \\
\cline { 2 - 3 } & $\begin{array}{l}\text { D. } N>M_{1}^{1}, N>M_{1}^{2} \text { and } \\
M_{1}^{1}+M_{2}^{1}+M_{1}^{2}+M_{2}^{2} \geq 3 N\end{array}$ & SSA for both clusters \\
\hline
\end{tabular}

TABLE II

TWo-USER CASE: $D o F^{*}=2\left(M_{2}^{1}+M_{2}^{2}\right)$

\begin{tabular}{|c|c|c|c|}
\hline \multicolumn{3}{|c|}{ Conditions when optimal $D o F^{*}$ can be achieved } & Optimal Strategy \\
\hline \multicolumn{3}{|c|}{ III. $N \geq 2\left(M_{2}^{1}+M_{2}^{2}\right)$} & $\mathrm{MAC}+\mathrm{BC}$ \\
\hline \multirow{8}{*}{$\begin{array}{l}\text { IV. } M_{2}^{1}+M_{2}^{2}<N \\
<2\left(M_{2}^{1}+M_{2}^{2}\right)\end{array}$} & A. $N \leq M$ & and $N \leq M_{1}^{2}$ & SSA for both clusters \\
\hline & \multirow{2}{*}{ B. $M_{1}^{1}<N \leq M_{1}^{2}$} & 1. $N \geq 2 M_{2}^{1}+M_{2}^{2}$ & $\begin{array}{l}\text { Cluster1: MAC+BC } \\
\text { Cluster 2: SSA }\end{array}$ \\
\hline & & 2. $M_{1}^{1} \geq M_{2}^{1}+M_{2}^{2}$ & $\begin{array}{l}\text { Relay uses } M_{2}^{1}+M_{2}^{2} \text { ant } \\
\text { with SSA at both clusters }\end{array}$ \\
\hline & \multirow{2}{*}{ C. $M_{1}^{2}<N \leq M_{1}^{1}$} & 1. $N \geq M_{2}^{1}+2 M_{2}^{2}$ & $\begin{array}{l}\text { Cluster1: SSA } \\
\text { Cluster2: MAC+BC }\end{array}$ \\
\hline & & 2. $M_{1}^{2} \geq M_{2}^{1}+M_{2}^{2}$ & $\begin{array}{l}\text { Relay uses } M_{2}^{1}+M_{2}^{2} \text { ant } \\
\text { with SSA at both clusters }\end{array}$ \\
\hline & \multirow{3}{*}{$\begin{array}{l}\text { D. } N>M_{1}^{1} \\
N>M_{1}^{2}\end{array}$} & $\begin{array}{l}\text { 1. } M_{1}^{1} \geq M_{2}^{1}+M_{2}^{2} \\
\text { and } \\
M_{1}^{2} \geq M_{2}^{1}+M_{2}^{2}\end{array}$ & $\begin{array}{l}\text { Relay uses } M_{2}^{1}+M_{2}^{2} \text { ant } \\
\text { with SSA at both clusters }\end{array}$ \\
\hline & & 2. $M_{1}^{2} \geq 2 M_{2}^{1}+M_{2}^{2}$ & $\begin{array}{l}\text { Relay uses } 2 M_{2}^{1}+M_{2}^{2} \text { ant } \\
\text { with Cluster1 MAC+BC } \\
\text { Cluster2 SSA }\end{array}$ \\
\hline & & 3. $M_{1}^{1} \geq M_{2}^{1}+2 M_{2}^{2}$ & $\begin{array}{l}\text { Relay uses } M_{2}^{1}+2 M_{2}^{2} \text { ant } \\
\text { with Cluster1 SSA } \\
\text { Cluster2 MAC+BC }\end{array}$ \\
\hline
\end{tabular}

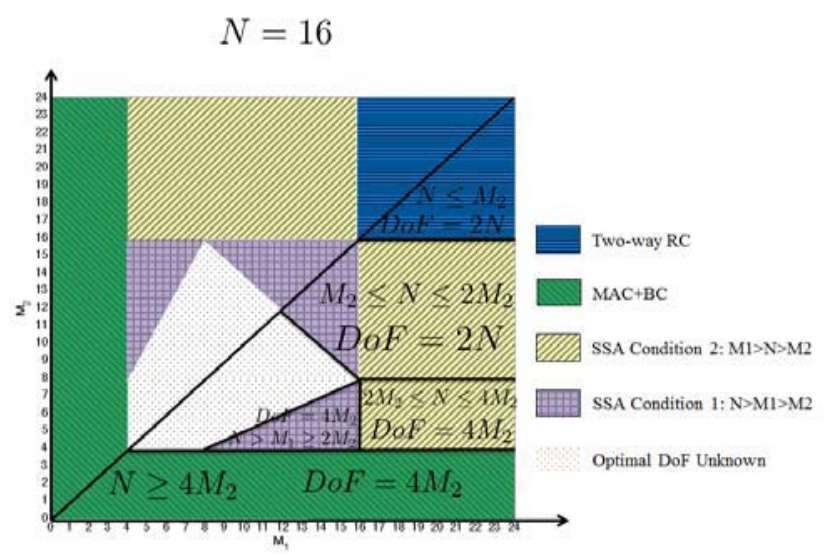

Fig. 5. Illustration for cases when DoF upper bound can be achieved.

alignment, where different SSA conditions depend on the number of antennas at the users and the relay. Note that the different SSA conditions correspond to those introduced in Lemma 1.

\section{Three Users in Each Cluster: General Case}

We now study the case when there are three users in each cluster for the general setting. Without loss of generality, assume $M_{1}^{1} \geq M_{2}^{1} \geq M_{3}^{1}$ and $M_{1}^{2} \geq M_{2}^{2} \geq M_{3}^{2}$.

Theorem 3: Consider the two-cluster MIMO multi-way relay channel with three users in each cluster. The optimal DoF and the achievable strategies are:

(1) $D o F^{*}=2 N$ See Table III.

(2) $D o F^{*}=M_{1}^{1}+M_{2}^{1}+M_{3}^{1}+M_{1}^{2}+M_{2}^{2}+M_{3}^{2}$ See Table IV.

(3) $D o F^{*}=2\left(M_{2}^{1}+M_{3}^{1}+M_{2}^{2}+M_{3}^{2}\right)$ See Table V.

(4) $D o F^{*}=M_{1}^{1}+M_{2}^{1}+M_{3}^{1}+2\left(M_{2}^{2}+M_{3}^{2}\right)$ See Table VI.

(5) $D o F^{*}=2\left(M_{2}^{1}+M_{3}^{1}\right)+M_{1}^{2}+M_{2}^{2}+M_{3}^{2}$ See Table VII.

Proof: See Appendix C for details of the proof and the achievable strategies.

\section{L-Cluster $K$-User MiMO Multi-WAY RELAY CHANNEL}

Consider now the general $L$-cluster $K$-user MIMO multiway relay channel in the symmetric setting, i.e., all the users have the same number of antennas. We have the following optimal DoF result. 
TABLE III

Three-User Case: $D o F^{*}=2 N$

\begin{tabular}{|l|l||l|}
\hline \multicolumn{2}{|l|}{ Conditions for optimal $D o F^{*}$} & Optimal Strategy \\
\hline \multicolumn{1}{|l|}{ I. $N \leq \max \left\{M_{2}^{1}, M_{2}^{2}\right\}$} & Two-way RC with FDF \\
\hline & A. $N \leq M_{1}^{1}$ and $N \leq M_{1}^{2}$ & SSA for both clusters \\
\cline { 2 - 3 } & B. $M_{1}^{2}<N \leq M_{1}^{1}$ and & \multirow{1}{*}{ SSA for both clusters } \\
& $M_{2}^{1}+M_{3}^{1}+\left(M_{2}^{1}+M_{3}^{1}-N\right)^{+}$ & \\
& $+\left(M_{1}^{2}+M_{2}^{2}-N\right)^{+}+\left(M_{1}^{2}+M_{3}^{2}-N\right)^{+}$ & \\
& $+\left(M_{2}^{2}+M_{3}^{2}-N\right)^{+} \geq N$ & \\
\cline { 2 - 3 } II. $N>$ & C. $M_{1}^{1}<N \leq M_{1}^{2}$ and & SSA for both clusters \\
$\max \left\{M_{2}^{1}, M_{2}^{2}\right\}$ & $\left(M_{1}^{1}+M_{2}^{1}-N\right)^{+}+\left(M_{1}^{1}+M_{3}^{1}-N\right)^{+}$ & \\
& $+\left(M_{2}^{1}+M_{3}^{1}-N\right)^{+}+M_{2}^{2}$ & \\
& $+M_{3}^{2}+\left(M_{2}^{2}+M_{3}^{2}-N\right)^{+} \geq N$ & \\
\cline { 2 - 3 } & D. $M_{1}^{1}<N, M_{1}^{2}<N$ and & \\
& $\left(M_{1}^{1}+M_{2}^{1}-N\right)^{+}+\left(M_{1}^{1}+M_{3}^{1}-N\right)^{+}$ & SSA for both clusters \\
& $+\left(M_{2}^{1}+M_{3}^{1}-N\right)^{+}+\left(M_{1}^{2}+M_{2}^{2}-N\right)^{+}$ & \\
& $+\left(M_{1}^{2}+M_{3}^{2}-N\right)^{+}+\left(M_{2}^{2}+M_{3}^{2}-N\right)^{+} \geq N$ & \\
\hline
\end{tabular}

TABLE IV

Three-USER CASE: $D o F^{*}=M_{1}^{1}+M_{2}^{1}+M_{3}^{1}+M_{1}^{2}+M_{2}^{2}+M_{3}^{2}$

\begin{tabular}{|l||l|}
\hline Conditions for optimal $D o F^{*}$ & Optimal Strategy \\
\hline III. $N \geq M_{1}^{1}+M_{2}^{1}+M_{3}^{1}+M_{1}^{2}+M_{2}^{2}+M_{3}^{2}$ & MAC $+\mathrm{BC}$ \\
\hline
\end{tabular}

TABLE V

Three-User CASE: $D o F^{*}=2\left(M_{2}^{1}+M_{3}^{1}+M_{2}^{2}+M_{3}^{2}\right)$

\begin{tabular}{|c|c|c|}
\hline \multicolumn{2}{|c|}{ Conditions for optimal $D o F^{*}$} & Optimal Strategy \\
\hline IV. $N \geq$ & $\left.M_{2}^{1}+M_{3}^{1}+M_{2}^{2}+M_{3}^{2}\right)$ & $\mathrm{MAC}+\mathrm{BC}$ \\
\hline \multirow{3}{*}{$\begin{array}{l}\text { V. } N<2\left(M_{2}^{1}+M_{3}^{1}\right. \\
\left.+M_{2}^{2}+M_{3}^{2}\right)\end{array}$} & $\begin{array}{l}\text { A. } M_{1}^{1} \geq M_{2}^{1}+M_{3}^{1}+M_{2}^{2}+M_{3}^{2} \\
M_{1}^{2} \geq M_{2}^{1}+M_{3}^{1}+M_{2}^{2}+M_{3}^{2}\end{array}$ & $\begin{array}{l}\# \text { antenna used at relay: } \\
M_{2}^{1}+M_{3}^{1}+M_{2}^{2}+M_{3}^{2} \\
\text { SSA at both clusters }\end{array}$ \\
\hline & $\begin{array}{l}\text { B. } N \geq 2\left(M_{2}^{1}+M_{3}^{1}\right)+M_{2}^{2}+M_{3}^{2} \\
M_{1}^{2} \geq 2\left(M_{2}^{1}+M_{3}^{1}\right)+M_{2}^{2}+M_{3}^{2}\end{array}$ & $\begin{array}{l}\# \text { antenna used at relay: } \\
2\left(M_{2}^{1}+M_{3}^{1}\right)+M_{2}^{2}+M_{3}^{2} ; \\
\text { Cluster1 MAC+BC; } \\
\text { Cluster2 SSA }\end{array}$ \\
\hline & $\begin{array}{l}\text { C. } N \geq M_{2}^{1}+M_{3}^{1}+2\left(M_{2}^{2}+M_{3}^{2}\right) \\
M_{1}^{1} \geq M_{2}^{1}+M_{3}^{1}+2\left(M_{2}^{2}+M_{3}^{2}\right)\end{array}$ & $\begin{array}{l}\# \text { antenna used at relay: } \\
M_{2}^{1}+M_{3}^{1}+2\left(M_{2}^{2}+M_{3}^{2}\right) \\
\text { Cluster1 SSA; } \\
\text { Cluster2 MAC+BC }\end{array}$ \\
\hline
\end{tabular}

TABLE VI

THREe-USER CASE: $D o F^{*}=M_{1}^{1}+M_{2}^{1}+M_{3}^{1}+2\left(M_{2}^{2}+M_{3}^{2}\right)$

\begin{tabular}{|l||l|}
\hline Conditions for optimal $D o F^{*}$ & Optimal Strategy \\
\hline VI. $N \geq M_{1}^{1}+M_{2}^{1}+M_{3}^{1}+2\left(M_{2}^{2}+M_{3}^{2}\right)$ & MAC+BC \\
\hline VII. $N \geq M_{1}^{1}+M_{2}^{1}+M_{3}^{1}+M_{2}^{2}+M_{3}^{2}$ & \# antennas used at the relay: \\
$N<M_{1}^{1}+M_{2}^{1}+M_{3}^{1}+2\left(M_{2}^{2}+M_{3}^{2}\right)$ & $M_{1}^{1}+M_{2}^{1}+M_{3}^{1}+M_{2}^{2}+M_{3}^{2} ;$ \\
$M_{1}^{2} \geq M_{1}^{1}+M_{2}^{1}+M_{3}^{1}+M_{2}^{2}+M_{3}^{2}$ & Cluster1 MAC+BC; \\
\hline
\end{tabular}

Theorem 4: For the symmetric $L$-cluster $K$-user MIMO multi-way relay channel, where all users have $M$ antennas and the relay has $N$ antennas, the optimal DoF is

$$
\begin{aligned}
& D o F^{*}=K L M \quad \text { if } N \geq K L M, \\
& D o F^{*}=2 N \quad \text { if } \frac{L K(K-1)}{2}(2 M-N) \geq N .
\end{aligned}
$$

To establish the optimal DoF, we first study the DoF upperbound. For this case, the DoF upperbound in equation (13) becomes

$$
D o F \leq \min \{K L M, 2 N\} .
$$

To investigate the achievability of the DoF upperbound, we further consider the following cases: 
TABLE VII

ThreE-USER CASE: $D o F^{*}=2\left(M_{2}^{1}+M_{3}^{1}\right)+M_{1}^{2}+M_{2}^{2}+M_{3}^{2}$

\begin{tabular}{|l||l|}
\hline Conditions for optimal $D o F^{*}$ & Optimal Strategy \\
\hline VIII. $N \geq 2\left(M_{2}^{1}+M_{3}^{1}\right)+M_{1}^{2}+M_{2}^{2}+M_{3}^{2}$ & MAC+BC \\
\hline IX. $N \geq M_{2}^{1}+M_{3}^{1}+M_{1}^{2}+M_{2}^{2}+M_{3}^{2}$ & $\#$ antennas used at the relay: \\
$N<2\left(M_{2}^{1}+M_{3}^{1}\right)+M_{1}^{2}+M_{2}^{2}+M_{3}^{2}$ & $M_{2}^{1}+M_{3}^{1}+M_{1}^{2}+M_{2}^{2}+M_{3}^{2} ;$ \\
$M_{1}^{1} \geq M_{2}^{1}+M_{3}^{1}+M_{1}^{2}+M_{2}^{2}+M_{3}^{2}$ & Cluster1 SSA; \\
\hline
\end{tabular}

\section{A. Achieving DoF K LM: Multiple-Access Transmission}

When $2 N>K L M$, the DoF upper bound becomes $K L M$. The DoF upper bound can be achieved when $N \geq K L M$. Under this condition, the relay can decode all the messages from all the users and can broadcast the messages to the intended users without inducing any interference.

\section{B. Achieving DoF 2N: Signal Space Alignment}

When $2 N \leq K L M$, the DoF upper bound becomes $2 N$. To achieve this upperbound, we require each signal dimension at the relay to be shared by a pair of users. From Lemma 1, any pair of users in the same cluster can share $2 M-N$ dimensional signal space at the relay, if $2 M \geq N$. Therefore, we need

$$
L\left(\begin{array}{l}
K \\
2
\end{array}\right)(2 M-N) \geq N,
$$

or equivalently

$$
\frac{L K(K-1)}{2}(2 M-N) \geq N,
$$

such that all the signal dimension at the relay can be shared by a pair of users. We can choose any pair of users to exchange data streams without exceeding their maximum allowed dimension of shared signal space $2 M-N$. We let the users exchange $N$ pairs of data streams, and the relay can decode the sum of each pair of data stream and broadcast to the users with proper receiver-side processing. The detailed transmission scheme is described as follows:

If $n=\frac{2 N}{L K(K-1)}$ is an integer, we let user $i$ and user $j$, $i, j=1,2, \ldots, K$ in cluster $l, l=1,2, \ldots, L$ exchange $n$ data streams, each with unit DoF. Since we have $2 M-N \geq n$, based on Lemma 1, each pair of users can transmit the data streams along the vectors $\mathbf{v}_{(i j), m}^{l}$ and $\mathbf{v}_{(j i), m}^{l}, m=1,2, \ldots, n$ such that

$$
\mathbf{H}_{R(i, l)} \mathbf{v}_{(i j), m}^{l}=\mathbf{H}_{R(j, l)} \mathbf{v}_{(j i), m}^{l}=\mathbf{q}_{(i j), m}^{l},
$$

where $\mathbf{v}_{(i j), m}^{l}$ denotes the $m$ th beamforming vector for user $i$ in cluster $l$ to share the signal space at the relay with user $j$ in cluster $l$.

The transmitted signal from user $i$ in cluster $l$ is thus

$$
\mathbf{X}_{i, l}=\sum_{\substack{j=1 \\ j \neq i}}^{K} \sum_{m=1}^{n} \mathbf{v}_{(i j), m}^{l} d_{(i j), m}^{l},
$$

where $d_{(i j), m}^{l}$ denotes the $m$ th message from user $i$ in cluster $l$ for user $j$ in cluster $l$.
The received signal at the relay is

$$
\begin{aligned}
\mathbf{Y}_{R} & =\sum_{l=1}^{L} \sum_{i=1}^{K} \mathbf{H}_{R(i, l)} \mathbf{X}_{i, l} \\
& =\sum_{l=1}^{L} \sum_{i=1}^{K} \sum_{\substack{j=1 \\
j \neq i}}^{K} \sum_{m=1}^{n} \mathbf{H}_{R(i, l)} \mathbf{v}_{(i j), m}^{l} d_{(i j), m}^{l} \\
& =\sum_{l=1}^{L} \sum_{i=1}^{K} \sum_{\substack{j=i+1 \\
j \neq i}}^{K} \sum_{m=1}^{n} \mathbf{q}_{(i j), m}^{l}\left(d_{(i j), m}^{l}+d_{(j i), m}^{l}\right) .
\end{aligned}
$$

The relay can decode $d_{(i j), m}^{l}+d_{(j i), m}^{l}$ and then need to broadcast the messages back to the users. Following a similar scheme as in the two-cluster case, we let user $i$ and user $j$ employ a receiver-side filter $\left(\mathbf{u}_{(i j), m}^{l}\right)$ and $\left(\mathbf{u}_{(j i), m}^{l}\right)$ to decode the message $d_{(i j), m}^{l}+d_{(j i), m}^{l}$, where

$$
\left(\mathbf{u}_{(i j), m}^{l}\right)^{T} \mathbf{H}_{(i, l) R}=\left(\mathbf{u}_{(j i), m}^{l}\right)^{T} \mathbf{H}_{R(j, l)}=\mathbf{g}_{(i j), m}^{l} .
$$

Finding the receiver-side filter is a dual problem to finding the beamforming vector $\mathbf{v}_{(i j), m}^{l}$, which can be seen by taking transpose of equation (42). Based on Lemma 1, there exist $2 M-N$ such pair of vectors $\left(\mathbf{u}_{(i j), m}^{l}\right)^{T}$ and $\left(\mathbf{u}_{(j i), m}^{l}\right)^{T}$. User $i$ in cluster $l$ can choose $n$ out of these vectors to form a filtering matrix $\mathbf{U}_{(i j)}^{l}$ to receive the messages $d_{(i j), m}^{l}+d_{T}^{l}(j i), m$, where the matrix $\mathbf{U}_{(i j)}^{l}$ is formed by taking $\left(\mathbf{u}_{(i j), m}^{l}\right)^{T}$ as its rows. We can also combine the matrices $\mathbf{U}_{(i j)}^{l}$ for all $j=1, \ldots, K$, $j \neq i$ to form the filtering matrix for user $i$ to decode all the intended messages:

$$
\mathbf{U}_{i}^{l}=\left[\begin{array}{c}
\mathbf{U}_{(i 1)}^{l} \\
\vdots \\
\mathbf{U}_{(i, i-1)}^{l} \\
\mathbf{U}_{(i, i+1)}^{l} \\
\vdots \\
\mathbf{U}_{(i K)}^{l}
\end{array}\right]
$$

The relay can use zero-forcing to broadcast the messages $d_{(i j), m}^{l}+d_{(j i), m}^{l}$ to the intended users. The users can decode the intended messages using their side information. The DoF $2 N$ is thus achievable.

When $n=\frac{2 N}{L K(K-1)}$ is not an integer, we can let one pair of users to exchange

$$
N-\left(\frac{L K(K-1)}{2}-1\right)\left\lceil\frac{2 N}{L K(K-1)}\right\rceil
$$


messages, and the other user pairs exchange

$$
\left\lceil\frac{2 N}{L K(K-1)}\right\rceil
$$

messages, and the DoF $2 N$ is still achievable.

Remark 4: Theorem 4 provides us with the first DoF result for the $L$-cluster, $K$-user MIMO multi-way relay channel for arbitrary $L, K$. We can see that the DoF is always limited by the available spatial dimension at the relay, and that with fixed number of antennas at the relay, increasing the number of users and the number of clusters cannot provide DoF gain. In addition, we gain the insight that the DoF optimal way to utilize the resources of the relay is to share the relay between two users. We cannot obtain DoF gain by letting three or more users to share the resources of the relay.

Remark 5: The result for the asymmetric case of the general $L$-cluster $K$-user MIMO multi-way relay channel can be obtained following similar arguments as in the two-cluster case. Other than having to enumerate a number of cases and conditions, the results do not provide further insights to what we already provide for the symmetric case. Hence the detailed expressions for the asymmetric cases are omitted here.

\section{CONCLUSION}

In this paper, we have investigated the DoF for the general MIMO multi-way relay channel and established the optimal DoF for several scenarios of interests. We have derived a new DoF upper bound using genie-aided approach, which is shown to be tight for several scenarios of interests. Specifically, we have studied the DoF for the two-cluster twouser MIMO multi-way relay channel and two-cluster threeuser MIMO multi-way relay channel with arbitrary number of antennas, and established the optimal DoF using signal space alignment, multiple-access transmission, or a combination of both, depending on the number of antennas at the users and the relay. We have also studied the $L$-cluster $K$-user MIMO multi-way relay channel with equal number of antennas at the users, and established the optimal DoF. The DoF results imply that the DoF of the MIMO multi-way relay channel is always limited by the spatial dimension available at the relay. With fixed number of antennas at the relay, increasing the number of users and clusters cannot provide any DoF gain. The results also imply that allowing three or more users to share the resources of the relay cannot provide any DoF gain.

This work has established the optimal DoF for a variety of scenarios for the multi-way relay channel which was unknown previously. For the remaining cases, determining the strategies to achieve the optimal DoF remains open.

\section{ACKNOWLEDGMENT}

The authors would like to thank the editor and anonymous reviewers of this work for their valuable comments and suggestions to improve the quality of this paper.

\section{APPENDIX A}

\section{PROOF OF LEMMA 1}

Proof: We first consider the case when $p \geq q_{1} \geq q_{2}$ and $q_{1}+q_{2}>p$. Note that equation (30) is equivalent as

$$
\left[\begin{array}{ccc}
\mathbf{I} & -\mathbf{H}_{1} & \mathbf{0} \\
\mathbf{I} & \mathbf{0} & -\mathbf{H}_{2}
\end{array}\right]\left[\begin{array}{c}
\mathbf{v}_{i} \\
\mathbf{u}_{i} \\
\mathbf{w}_{i}
\end{array}\right]=\mathbf{0} .
$$

The null space of the matrix

$$
\left[\begin{array}{ccc}
\mathbf{I} & -\mathbf{H}_{1} & \mathbf{0} \\
\mathbf{I} & \mathbf{0} & -\mathbf{H}_{2}
\end{array}\right]
$$

has dimension $q_{1}+q_{2}-p$. It is easy to see that if $q_{1}+q_{2}>p$, then we can find $q_{1}+q_{2}-p$ non-zero linearly independent vectors of the form

$$
\left[\begin{array}{lll}
\mathbf{v}_{i}^{T} & \mathbf{u}_{i}^{T} & \mathbf{w}_{i}^{T}
\end{array}\right]^{T}
$$

from the null space of the matrix shown in equation (47). It remains to see whether all these vectors satisfy $\mathbf{v}_{i} \neq 0$. Since $p \geq q_{1} \geq q_{2}$, we can see that the null space of matrices $\mathbf{H}_{1}$ and $\mathbf{H}_{2}$ has dimension 0. Therefore for all the non-zero vectors satisfying equation (46), we must have $\mathbf{v}_{i} \neq 0$.

Similarly, when $q_{1} \geq p \geq q_{2}$, we can find $q_{1}+q_{2}-p$ non-zero linearly independent vectors of the form shown in equation (48) to satisfy equation (46). However, for this case, if we consider the equation $\mathbf{v}_{i}=\mathbf{H}_{2} \mathbf{w}_{i}$, we can see that there are at most $q_{2}$ non-zero linearly independent vectors $\mathbf{v}_{i}$ satisfying this equation. In fact, since $q_{1} \geq p$, the null space of matrix $\mathbf{H}_{1}$ has dimension $q_{1}-p$. When we set $\mathbf{w}_{i}$ and $\mathbf{v}_{i}$ to 0 , we can find $q_{1}-p$ non-zero linearly independent vectors $\mathbf{u}_{i}$ to satisfy equation (46). We can construct the vectors $\mathbf{u}_{i}$ as

$$
\mathbf{u}_{i}=\mathbf{H}_{1}^{*}\left(\mathbf{H}_{1} \mathbf{H}_{1}^{*}\right)^{-1} \mathbf{H}_{2} \mathbf{w}_{i} .
$$

Therefore we can conclude that among all vectors of the form in equation (48) satisfying equation (46), we can only find $q_{2}$ non-zero linearly independent vectors $\mathbf{v}_{i}$.

\section{APPENDIX B}

\section{Two-User Two-Cluster Multi-Way Relay Channel: Transmission Strategies}

For the two-cluster multi-way relay channel with two users in each cluster, we can show that the following DoF is achievable:

Proposition 1: (i) When $N \leq M_{2}^{1}+M_{2}^{2}$, the following DoF is achievable:

- Case 1: $N \leq M_{2}^{1}$, DoF $=2 N$.

- Case 2: $N>M_{2}^{1} \geq M_{2}^{2}$

- Condition 1: $N \leq M_{1}^{1}$ and $N \leq M_{1}^{2}, D o F=2 N$.

- Condition 2: $M_{1}^{1}<N \leq M_{1}^{2}$

1) $M_{1}^{1}+M_{2}^{1}+M_{2}^{2} \geq 2 N, D o F=2 N$.

2) $M_{1}^{1}+M_{2}^{1}+M_{2}^{2}<2 N$, $D o F=M_{1}^{1}+M_{2}^{1}+M_{2}^{2}$.

- Condition 3: $M_{1}^{2}<N \leq M_{1}^{1}$

1) $M_{2}^{1}+M_{1}^{2}+M_{2}^{2} \geq 2 N, D o F=2 N$.

2) $M_{2}^{1}+M_{1}^{2}+M_{2}^{2}<2 N, D o F=\max \left\{M_{2}^{1}+M_{1}^{2}+\right.$ $\left.M_{2}^{2}, N+M_{2}^{1}\right\}$. 
- Condition 4: $N>M_{1}^{1}, N>M_{1}^{2}$

1) $M_{1}^{1}+M_{2}^{1}+M_{1}^{2}+M_{2}^{2} \geq 3 N, D o F=2 N$.

2) $M_{1}^{1}+M_{2}^{1}+M_{1}^{2}+M_{2}^{2}<3 N$,

$$
\begin{aligned}
D o F=\max \{ & \left(M_{1}^{2}+M_{2}^{2}-N\right)^{+}+N, \\
& M_{1}^{1}+M_{2}^{1}, \\
& \min \left\{\frac{2\left(M_{1}^{1}+M_{2}^{1}+M_{1}^{2}+M_{2}^{2}\right)}{3},\right. \\
& \frac{4\left(M_{1}^{1}+M_{2}^{1}+M_{2}^{2}\right)-2 M_{1}^{2}}{3}, \\
& \left.\left.\frac{4\left(M_{2}^{1}+M_{1}^{2}+M_{2}^{2}\right)-2 M_{1}^{1}}{3}\right\}\right\} .
\end{aligned}
$$

(ii) When $N>M_{2}^{1}+M_{2}^{2}$, the following DoF is achievable:

- Case 1: $N \geq 2\left(M_{2}^{1}+M_{2}^{2}\right)$, DoF $=2\left(M_{2}^{1}+M_{2}^{2}\right)$.

- Case 2: $N<2\left(M_{2}^{1}+M_{2}^{2}\right) \leq 2\left(M_{2}^{1}+M_{2}^{1}\right) \leq 2\left(M_{1}^{1}+M_{2}^{1}\right)$

- Condition 1: $N \leq M_{1}^{1}$ and $N \leq M_{1}^{2}$, DoF $=2\left(M_{2}^{1}+\right.$ $\left.M_{2}^{2}\right)$.

- Condition 2: $M_{1}^{1}<N \leq M_{1}^{2}$, which implies $M_{1}^{1}+$ $M_{2}^{1}+M_{2}^{2}<2 N$,

1) $N \geq 2 M_{2}^{1}+M_{2}^{2}, D o F=2\left(M_{2}^{1}+M_{2}^{2}\right)$.

2) $M_{1}^{1} \geq M_{2}^{1}+M_{2}^{2}, D o F=2\left(M_{2}^{1}+M_{2}^{2}\right)$.

3) $N<2 M_{2}^{1}+M_{2}^{2}$ and $M_{1}^{1}<M_{2}^{1}+M_{2}^{2}$, DoF $=$ $\max \left\{N+M_{2}^{2}, M_{1}^{1}+M_{2}^{1}+M_{2}^{2}\right\}$.

- Condition 3: $M_{1}^{2}<N \leq M_{1}^{1}$, which implies $M_{2}^{1}+$ $M_{1}^{2}+M_{2}^{2}<2 N$,

1) $N \geq M_{2}^{1}+2 M_{2}^{2}, D o F=2\left(M_{2}^{1}+M_{2}^{2}\right)$.

2) $M_{1}^{2} \geq M_{2}^{1}+M_{2}^{2}$, $D o F=2\left(M_{2}^{1}+M_{2}^{2}\right)$.

3) $N<M_{2}^{1}+2 M_{2}^{2}$ and $M_{1}^{2}<M_{2}^{1}+M_{2}^{2}$, DoF= $\max \left\{M_{2}^{1}+M_{1}^{2}+M_{2}^{2}, N+M_{2}^{1}\right\}$.

- Condition 4: $N>M_{1}^{1}, N>M_{1}^{2}$, which implies $M_{1}^{1}+M_{2}^{1}+M_{1}^{2}+M_{2}^{2}<3 N$,

1) $M_{1}^{1} \geq M_{2}^{1}+M_{2}^{2}$ and $M_{1}^{2} \geq M_{2}^{1}+M_{2}^{2}$, DoF $=$ $2\left(M_{2}^{1}+M_{2}^{2}\right)$.

2) $M_{1}^{2} \geq 2 M_{2}^{1}+M_{2}^{2}, D o F=2\left(M_{2}^{1}+M_{2}^{2}\right)$.

3) $M_{1}^{1} \geq M_{2}^{1}+2 M_{2}^{2}, D o F=2\left(M_{2}^{1}+M_{2}^{2}\right)$.

4) Otherwise,

$$
\begin{aligned}
\text { DoF }=\max \{ & N,\left(M_{1}^{1}+M_{2}^{1}-N\right)^{+}+N, \\
& \left(M_{1}^{2}+M_{2}^{2}-N\right)^{+}+N \\
& \min \left\{\frac{2\left(M_{1}^{1}+M_{2}^{1}+M_{1}^{2}+M_{2}^{2}\right)}{3},\right. \\
& \frac{4\left(M_{1}^{1}+M_{2}^{1}+M_{2}^{2}\right)-2 M_{1}^{2}}{3}, \\
& \left.\left.\frac{4\left(M_{2}^{1}+M_{1}^{2}+M_{2}^{2}\right)-2 M_{1}^{1}}{3}\right\}\right\} .
\end{aligned}
$$

Proof: We next provide detailed transmission schemes to show how the above DoF can be achieved and identify scenarios when the DoF upper bound can be achieved.

(i) When $N \leq M_{2}^{1}+M_{2}^{2}$ :

Under this condition, the DoF upper bound in equation (13) reduces to

$$
D o F \leq 2 N \text {. }
$$

We further consider the following cases:

Case 1: $N \leq M_{2}^{1}$ : This condition corresponds to the case when the relay always has less antennas than both users in at least one of the two clusters. The DoF $2 N$ can be achieved by only allowing the users in the cluster with more antennas than the relay to exchange information, which yields a two-way relay channel. Since both users have more antennas than the relay, they can perfectly align $N$ independent data streams at the relay. The functional-decode-and-forward (FDF) strategy can thus achieve the DoF upper bound $2 N$.

Case 2: $N>M_{2}^{1} \geq M_{2}^{2}$ : This condition corresponds to the case when the relay has more antennas than at least one users in each cluster. A single pair of users thus cannot perfectly align $N$ independent data streams at the relay. However, it is still possible to achieve the optimal DoF by allowing two clusters of users to use the relay at the same time. Depending on the number of antennas at the relay and the users, we further consider the following conditions:

Condition 1: $N \leq M_{1}^{1}$ and $N \leq M_{1}^{2}$. For this case, one of the users in each cluster has more antennas than the relay. From Condition 2 in Lemma 1 , if we set $\mathbf{H}_{1}=\mathbf{H}_{R(1,1)}$ and $\mathbf{H}_{2}=\mathbf{H}_{R(2,1)}$, we can see that for user 1 and user 2 in cluster 1 , they can find $M_{2}^{1}$ non-zero linearly independent vectors $\mathbf{q}_{1 i}, \mathbf{v}_{(1,1) i}$ and $\mathbf{v}_{(2,1) i}, i=1, \ldots, M_{2}^{1}$ for cluster 1 such that

$$
\mathbf{H}_{R(1,1)} \mathbf{v}_{(1,1) i}=\mathbf{H}_{R(2,1)} \mathbf{v}_{(2,1) i}=\mathbf{q}_{1 i} .
$$

This means that user 1 and user 2 can share $M_{2}^{1}$ dimensional space at the relay. Following the same argument, we can see that user 1 and user 2 in cluster 2 share $M_{2}^{2}$ dimensional space at the relay, i.e., they can find $M_{2}^{2}$ non-zero linearly independent vectors $\mathbf{q}_{2 i}, \mathbf{v}_{(1,2) i}$ and $\mathbf{v}_{(2,2) i}$ such that

$$
\mathbf{H}_{R(1,2)} \mathbf{v}_{(1,2) i}=\mathbf{H}_{R(2,2)} \mathbf{v}_{(2,2) i}=\mathbf{q}_{2 i} \text {. }
$$

Since we have $M_{2}^{1}+M_{2}^{2} \geq N$, the users in cluster 1 can choose $M_{2}^{1 \prime}$ vectors out of the vectors $\mathbf{q}_{1 i}$, and the users in cluster 2 can choose $M_{2}^{2 \prime}$ vectors out of the vectors $\mathbf{q}_{2 i}$, such that these vectors are linearly independent almost surely and $M_{2}^{1 \prime}+M_{2}^{2 \prime}=N$, as their target signal directions at the relay. We denote the set of vectors chosen by cluster 1 as $\mathcal{Q}_{1}$ and the set of vectors chosen by cluster 2 as $\mathcal{Q}_{2}$.

Based on the above analysis, we can construct the transmission scheme as follows: User 1 and user 2 in cluster 1 send $M_{2}^{1 \prime}$ independent data streams $d_{1 i}^{1}$ and $d_{2 i}^{1}$ along the directions $\mathbf{v}_{(1,1) i}$ and $\mathbf{v}_{(2,1) i}$, respectively. User 1 and user 2 in cluster 2 send $M_{2}^{2 \prime}$ independent data streams $d_{1 i}^{2}$ and $d_{2 i}^{2}$ along the directions $\mathbf{v}_{(1,2) i}$ and $\mathbf{v}_{(2,2) i}$, respectively. We have

$$
\mathbf{X}_{k, 1}=\sum_{i \in \mathcal{Q}_{1}} \mathbf{v}_{(k, 1) i} d_{k i}^{1}, k=1,2,
$$




$$
\mathbf{X}_{k, 2}=\sum_{i \in \mathcal{Q}_{2}} \mathbf{v}_{(k, 2) i} d_{k i}^{2}, k=1,2 .
$$

The received signal at the relay is

$$
\begin{aligned}
\mathbf{Y}_{R} & =\sum_{k=1}^{2} \mathbf{H}_{R(k, 1)} \mathbf{X}_{k, 1}+\sum_{k=1}^{2} \mathbf{H}_{R(k, 2)} \mathbf{X}_{k, 2} \\
& =\sum_{i \in \mathcal{Q}_{1}} \mathbf{q}_{1 i}\left(d_{1 i}^{1}+d_{2 i}^{1}\right)+\sum_{i \in \mathcal{Q}_{2}} \mathbf{q}_{2 i}\left(d_{1 i}^{2}+d_{2 i}^{2}\right)
\end{aligned}
$$

The relay can then decode $d_{1 i}^{1}+d_{2 i}^{1}$ and $d_{1 i}^{2}+d_{2 i}^{2}$ using zero forcing.

The relay now needs to transmit $d_{1 i}^{1}+d_{2 i}^{1}$ to user 1 and user 2 in cluster 1 and also transmit $d_{1 i}^{2}+d_{2 i}^{2}$ to user 1 and user 2 in cluster 2 . For this end, we let the users apply a receiver-side filter $\mathbf{u}_{(k, l) i}$ such that

$$
\begin{aligned}
& \left(\mathbf{u}_{(1,1) i}\right)^{T} \mathbf{H}_{(1,1) R}=\left(\mathbf{u}_{(2,1) i}\right)^{T} \mathbf{H}_{(2,1) R}=\mathbf{g}_{1 i}^{T}, \\
& \left(\mathbf{u}_{(1,2) i}\right)^{T} \mathbf{H}_{(1,2) R}=\left(\mathbf{u}_{(2,2) i}\right)^{T} \mathbf{H}_{(2,2) R}=\mathbf{g}_{2 i}^{T},
\end{aligned}
$$

which makes the users in one cluster appear to be the same user to the relay.

Taking transpose of equations (59) and (60), we can see that the problem of finding the vectors $\mathbf{u}_{(k, l) i}$ are the same problem as finding the vectors $\mathbf{v}_{(k, l) i}$. Therefore the users in cluster $l$ can find $M_{2}^{l}$ such triplets of non-zero linearly independent vectors

$$
\left(\mathbf{u}_{(1, l) i}, \mathbf{u}_{(2, l) i}, \mathbf{g}_{l i}\right) .
$$

The users in cluster 1 can then choose $M_{2}^{1 \prime}$ vectors $\mathbf{g}_{1 i}$ and the users in cluster 2 can choose $M_{2}^{2 \prime}$ vectors $\mathbf{g}_{2 i}$, such that they are all linearly independent, as their target directions to receive signals transmitted from the relay. Using these chosen vectors, user $k$ in cluster $l$ can form a beamforming matrix $\mathbf{U}_{k, l}$, which has the chosen $\mathbf{u}_{(k, l) i}$ vectors as its rows, and apply it to the received signals:

$$
\begin{aligned}
\mathbf{Y}_{k, l}^{\prime} & =\mathbf{U}_{k, l} \mathbf{Y}_{k, l} \\
& =\mathbf{U}_{k, l} \mathbf{H}_{(k, l) R} \mathbf{X}_{R}+\mathbf{U}_{k, l} \mathbf{Z}_{k, l} \\
& =\mathbf{G}_{l} \mathbf{X}_{R}+\mathbf{U}_{k, l} \mathbf{Z}_{k, l}
\end{aligned}
$$

where the matrix $\mathbf{G}_{l}$ is of dimension $M_{2}^{l^{\prime}} \times N$ and has the chosen vectors $\mathbf{g}_{l i}$ as its rows. The relay can use zero-forcing precoding to communicate $d_{1 i}^{1}+d_{2 i}^{1}$ and $d_{1 i}^{2}+d_{2 i}^{2}$ to the intended users. The users can now subtract their own side information from the received signals to decode the intended messages. Therefore the DoF $2 N$ is achievable.

Condition 2: $M_{1}^{1}<N \leq M_{1}^{2}$. For this case, cluster 2 has a user with more antennas than the relay while both users in cluster 1 have less antennas than the relay. From Condition 1 in Lemma 1, the users in cluster 1 can share $M_{1}^{1}+M_{2}^{1}-N$ dimensional signal space at the relay, and from Condition 2 in Lemma 1, the users in cluster 2 can share $M_{2}^{2}$ dimensional signal space at the relay. Note that since $N \leq M_{2}^{1}+M_{2}^{2}$ and we assume $M_{2}^{1} \geq M_{2}^{2}$, we have $N \leq M_{1}^{1}+M_{2}^{1}$, i.e., $M_{1}^{1}+M_{2}^{1}-N$ is always greater than zero. This leads to the following two cases that we need to investigate:
1) $M_{1}^{1}+M_{2}^{1}+M_{2}^{2} \geq 2 N$. For this case, the total dimension of the shared signal space for the two clusters exceeds the available dimension available at the relay. The transmission scheme for the case $N \leq M_{1}^{1}$ and $N \leq M_{1}^{2}$ can be used to achieve the DoF $2 N$. Note that for this case, user 1 in cluster 2 has more antennas than the relay, and therefore it can send signals targeted at any signal dimension at the relay. User 2 in cluster 2 can transmit its data streams using some random beamforming vectors, and user 1 in cluster 2 can control the direction of its transmitted data streams such that they arrive aligned with the data streams sent by user 2 in cluster 2. Users in cluster 1 , on the other hand, need to design their beamforming vectors jointly such that their data streams are aligned at the relay. The received data streams from cluster 1 and cluster 2 are linearly independent at the relay almost surely since the channel matrices are generated from a continuous distribution. The relay then decodes the sum of the messages from each clusters, and broadcasts the messages back to the intended clusters with proper receiverside filtering at the users. The detailed scheme is similar to the previous case and is thus omitted.

2) $M_{1}^{1}+M_{2}^{1}+M_{2}^{2}<2 N$. Under this condition, the signal space available at the relay cannot be fully utilized by the two clusters, because the total dimension of shared signal space for the two clusters is $M_{1}^{1}+M_{2}^{1}-N+M_{2}^{2}$, which is smaller than $N$. Therefore the DoF upper bound $2 N$ cannot be achieved using signal space alignment.

For this case, if $\frac{M_{1}^{1}+M_{2}^{1}+M_{2}^{2}}{2}$ is an integer, we can let the relay to use $N^{\prime}=\frac{M_{1}^{1}+M_{2}^{1}+M_{2}^{2}}{2}$ antennas to assist the users. It is easy to see that $N^{\prime} \geq M_{2}^{1}, M_{1}^{2} \geq N^{\prime} \geq M_{2}^{2}$, and $N^{\prime} \geq$ $M_{1}^{1}$ since $M_{1}^{1}<N \leq M_{2}^{1}+M_{2}^{2}$. By using only a subset of the antennas at the relay, users in cluster 1 can still share $M_{1}^{1}+M_{2}^{1}-N$ dimensional space and users in cluster 2 can still share $M_{2}^{2}$ dimensional space. Since we also have $M_{1}^{1}+M_{2}^{1}-$ $N+M_{2}^{2}=N^{\prime}$, using the schemes described in the previous part, we can achieve the DoF $M_{1}^{1}+M_{2}^{1}+M_{2}^{2}$.

If $\frac{M_{1}^{1}+M_{2}^{1}+M_{2}^{2}}{2}$ is not an integer, we can use a twosymbol extension to create an effectively two-cluster MIMO multi-way relay channel with $2 M_{1}^{1}, 2 M_{2}^{1}, 2 M_{1}^{2}, 2 M_{2}^{2}, 2 N$ antennas at the users and the relay, respectively, and using the same argument as in the case when $\frac{M_{1}^{1}+M_{2}^{1}+M_{2}^{2}}{2}$ is an integer, we can achieve the DoF $M_{1}^{1}+M_{2}^{1}+M_{2}^{2}$ per channel use.

Remark 6: Note that under this condition $M_{1}^{1}+M_{2}^{1}+M_{2}^{2}<$ $2 N$, an alternative scheme is to let the relay use $N$ antennas to assist the users. The users in cluster 2 can still share the $M_{2}^{2}$ dimensional signal space at the relay. The users in cluster 1 can use the shared $M_{1}^{1}+M_{2}^{1}-N$ dimensional space for signal space alignment, which yields an achievable DoF of $2\left(M_{1}^{1}+M_{2}^{1}+\right.$ $\left.M_{2}^{2}\right)-2 N$ or use the rest $N-M_{2}^{2} \leq M_{2}^{1}$ dimensional space in the multiple-access fashion, which yields an achievable DoF of $N+M_{2}^{2}$. It is easy to see that using signal space alignment with a subset of the relay's antennas, we can achieve larger DoF than using all antennas at the relay with signal space alignment and multiple-access type of schemes. Note that since we only have the achievable DoF for this case, there may exist schemes that can achieve larger DoF and improve upon our result using all antennas at the relay. 
Condition 3: $M_{1}^{2}<N \leq M_{1}^{1}$. Based on Lemma 1, the users in cluster 1 share a $M_{2}^{1}$ dimensional signal space at the relay and the users in cluster 2 share a $M_{1}^{2}+M_{2}^{2}-N$ dimensional signal space at the relay. Different from the case when $M_{1}^{1}<$ $N \leq M_{1}^{2}$, for users in cluster 2 , we cannot guarantee that $M_{1}^{2}+$ $M_{2}^{2}-N$ is always positive. We further investigate the following cases:

1) $M_{2}^{1}+M_{1}^{2}+M_{2}^{2} \geq 2 N$. For this case, the total dimension of the shared signal space of the two clusters exceeds the available dimension of the signal space at the relay. The DoF $2 N$ can thus be achieved using signal space alignment, as described in the scheme for Case 2 - Condition 2.(1), $N \leq M_{2}^{1}+M_{2}^{2}$.

2) $M_{2}^{1}+M_{1}^{2}+M_{2}^{2}<2 N$. This condition implies that

$$
M_{1}^{2}+M_{2}^{2}-N<\frac{M_{1}^{2}+M_{2}^{2}-M_{2}^{1}}{2} .
$$

When $M_{1}^{2}+M_{2}^{2} \leq M_{2}^{1}$, we have $M_{1}^{2}+M_{2}^{2}-N<0$, i.e., users in cluster 2 cannot share any signal space at the relay. Therefore we let users in cluster 1 use the shared $M_{2}^{1}$ dimensional signal space to perform signal space alignment, and let the users in cluster 2 use the rest $N-M_{2}^{1}$ dimensional signal space at the relay in the multiple-access fashion. After decoding the sum of the messages from cluster 1 and the individual messages from cluster 2, the relay can then use zero-forcing precoding to broadcast the messages to the intended users with proper receiver-side filtering at users in cluster 1 . Using this scheme, users in cluster 1 can exchange $2 M_{2}^{1}$ messages and the users in cluster 2 can exchange $N-M_{2}^{1}$ messages. We can achieve DoF $N+M_{2}^{1}$.

When $M_{1}^{2}+M_{2}^{2}>M_{2}^{1}, M_{1}^{2}+M_{2}^{2}-N$ can be positive. For this case, we can let the relay use $N^{\prime}=\frac{M_{2}^{1}+M_{1}^{2}+M_{2}^{2}}{2}$ antennas to assist the users. Since we have $M_{2}^{1}+M_{2}^{2} \geq N>M_{1}^{2}$, $N^{\prime} \geq M_{1}^{2} \geq M_{2}^{2}$. We also have $M_{1}^{1}>N^{\prime}>M_{2}^{1}$. Following the results in Case 2 - Condition 2.(2), $N \leq M_{2}^{1}+M_{2}^{2}$, we can achieve the DoF $M_{2}^{1}+M_{1}^{2}+M_{2}^{2}$.

We can also let the relay use all the antennas to assist the users. If we allow the users in both clusters to use signal space alignment, the achievable DoF is $2\left(M_{2}^{1}+M_{1}^{2}+M_{2}^{2}\right)-2 N$. It is easy to see that this achievable DoF is always smaller than $M_{2}^{1}+M_{1}^{2}+M_{2}^{2}$ under the condition $M_{2}^{1}+M_{1}^{2}+M_{2}^{2}<2 N$. We can also let the users in cluster 1 use signal space alignment, but the users in cluster 2 use the relay in the multipleaccess fashion. This yields the achievable DoF $N+M_{2}^{1}$.

Condition 4: $N>M_{1}^{1}$ and $N>M_{1}^{2}$. Based on Lemma 1, users in cluster 1 share a $M_{1}^{1}+M_{2}^{1}-N$ dimensional signal space, and users in cluster 2 share a $M_{1}^{2}+M_{2}^{2}-N$ dimensional signal space at the relay. Note that we always have $M_{1}^{1}+M_{2}^{1}-$ $N>0$ for $N \leq M_{2}^{1}+M_{2}^{2}$. We further investigate the following cases:

1) $M_{1}^{1}+M_{2}^{1}+M_{1}^{2}+M_{2}^{2} \geq 3 N$. For this case, the total dimension of shared signal space for the two clusters exceeds the available signal space at the relay. Both clusters can use signal space alignment to achieve the DoF upper bound $2 N$. The scheme can be designed in the same fashion as in previous cases and the details are thus omitted.
2) $M_{1}^{1}+M_{2}^{1}+M_{1}^{2}+M_{2}^{2}<3 N$. This condition implies that

$$
M_{1}^{2}+M_{2}^{2}-N<\frac{2\left(M_{1}^{2}+M_{2}^{2}\right)-\left(M_{1}^{1}+M_{2}^{1}\right)}{3} .
$$

When $2\left(M_{1}^{2}+M_{2}^{2}\right) \leq M_{1}^{1}+M_{2}^{1}, M_{1}^{2}+M_{2}^{2}-N$ is always less than zero, i.e., there is no shared signal space at the relay for the users in cluster 2. For this case, we let the relay use all the antennas to assist the users. Users in cluster 1 can always share the $M_{1}^{1}+M_{2}^{1}-N$ dimensional signal space at the relay. The users in cluster 2 use the relay in the multiple-access fashion. This yields the achievable DoF

$$
2\left(M_{1}^{1}+M_{2}^{1}-N\right)+N-\left(M_{1}^{1}+M_{2}^{1}-N\right)=M_{1}^{1}+M_{2}^{1} .
$$

When $2\left(M_{1}^{2}+M_{2}^{2}\right)>M_{1}^{1}+M_{2}^{1}, M_{1}^{2}+M_{2}^{2}-N$ can be positive. For this case, we let the relay use only $N^{\prime}=$ $\frac{M_{1}^{1}+M_{2}^{1}+M_{1}^{2}+M_{2}^{2}}{3}$ antennas to assist the users, if $\frac{M_{1}^{1}+M_{2}^{1}+M_{1}^{2}+M_{2}^{2}}{3}$ is an integer. The case when $\frac{M_{1}^{1}+M_{2}^{1}+M_{1}^{2}+M_{2}^{2}}{3}$ is not an integer can be addressed using symbol extension. It is easy to see that $N^{\prime}>M_{2}^{1}$ and $N^{\prime}>M_{2}^{2}$. However, the relation between $\frac{M_{1}^{1}+M_{2}^{1}+M_{1}^{2}+M_{2}^{2}}{3}$ and $M_{1}^{1}$ depends on the relation between $M_{2}^{1}+M_{1}^{2}+M_{2}^{2}$ and $2 M_{1}^{1}$; the relation between $\frac{M_{1}^{1}+M_{2}^{1}+M_{1}^{2}+M_{2}^{2}}{3}$ and $M_{1}^{2}$ depends on the relation between $M_{1}^{1}+M_{2}^{1}+M_{2}^{2}$ and $2 M_{1}^{2}$ :

- $M_{2}^{1}+M_{1}^{2}+M_{2}^{2} \geq 2 M_{1}^{1}$ and $M_{1}^{1}+M_{2}^{1}+M_{2}^{2} \geq 2 M_{1}^{2}$ : For this case, users in cluster 1 share $\frac{2\left(M_{1}^{1}+M_{2}^{1}\right)-\left(M_{1}^{2}+M_{2}^{2}\right)}{3}$ dimensional signal space and users in cluster 2 share $\frac{2\left(M_{1}^{2}+M_{2}^{2}\right)-\left(M_{1}^{1}+M_{2}^{1}\right)}{3}$ dimensional signal space. The achievable DoF is $\frac{2\left(M_{1}^{1}+M_{2}^{1}+M_{1}^{2}+M_{2}^{2}\right)}{3}$.

- $M_{2}^{1}+M_{1}^{2}+M_{2}^{2} \geq 2 M_{1}^{1}$ and $M_{1}^{1}+M_{2}^{1}+M_{2}^{2}<2 M_{1}^{2}$ :

For this case, users in cluster 1 share $\frac{2\left(M_{1}^{1}+M_{2}^{1}\right)-\left(M_{1}^{2}+M_{2}^{2}\right)}{3}$ dimensional signal space and users in cluster 2 share $M_{2}^{2}$ dimensional signal space. The achievable DoF is $\frac{4\left(M_{1}^{1}+M_{2}^{1}+M_{2}^{2}\right)-2 M_{1}^{2}}{3}$.

- $M_{2}^{1}+M_{1}^{2}+M_{2}^{2}<2 M_{1}^{1}$ and $M_{1}^{1}+M_{2}^{1}+M_{2}^{2} \geq 2 M_{1}^{2}$ : For this case, users in cluster 1 share $M_{2}^{1}$ dimensional signal space and users in cluster 2 share $\frac{2\left(M_{1}^{2}+M_{2}^{2}\right)-\left(M_{1}^{1}+M_{2}^{1}\right)}{3}$ dimensional signal space. The achievable DoF is $\frac{4\left(M_{2}^{1}+M_{1}^{2}+M_{2}^{2}\right)-2 M_{1}^{1}}{3}$.

- $M_{2}^{1}+M_{1}^{3}+M_{2}^{2}<2 M_{1}^{1}$ and $M_{1}^{1}+M_{2}^{1}+M_{2}^{2}<2 M_{1}^{2}$ : This case is not possible since the first condition implies $M_{1}^{1}>M_{1}^{2}$ and the second condition implies $M_{1}^{1}<M_{1}^{2}$.

From the above cases, we can see that the achievable DoF is

$$
\begin{aligned}
& \min \left\{\frac{2\left(M_{1}^{1}+M_{2}^{1}+M_{1}^{2}+M_{2}^{2}\right)}{3},\right. \\
& \frac{4\left(M_{1}^{1}+M_{2}^{1}+M_{2}^{2}\right)-2 M_{1}^{2}}{3}, \\
& \left.\frac{4\left(M_{2}^{1}+M_{1}^{2}+M_{2}^{2}\right)-2 M_{1}^{1}}{3}\right\}
\end{aligned}
$$

Note that we can also let relay use all the antennas to assist the users. We only allow cluster 1 to use signal space alignment, and let users in cluster 2 use the relay in the 
multiple-access fashion. This yields the achievable DoF $M_{1}^{1}+M_{2}^{1}$. In addition, we can also let cluster 1 use the relay in the multiple-access fashion, and cluster 2 use signal space alignment. This yields the achievable DoF $\left(M_{1}^{2}+M_{2}^{2}-N\right)^{+}+N$. Combining both achievable DoF, we have the desired result in equation (50).

Remark 7: Note that we can also use multiple-access transmission for both clusters. However, the achievable DoF $N$ is always less than $M_{1}^{1}+M_{2}^{1}$.

Remark 8: Similar to Remark 6, if we let the relay use all the antennas and use signal space alignment, the achievable $\mathrm{DoF}$ is $2\left(M_{1}^{1}+M_{2}^{1}-N\right)+2\left(M_{1}^{2}+M_{2}^{2}-N\right)^{+}$which is smaller than the achievable DoF in (50).

(ii) When $N>M_{2}^{1}+M_{2}^{2}$ :

Under this setting, the DoF upper bound in equation (13) reduces to

$$
D o F \leq 2\left(M_{2}^{1}+M_{2}^{2}\right) .
$$

Case 1: $N \geq 2\left(M_{2}^{1}+M_{2}^{2}\right)$

The DoF upper bound can be easily achieved for this case since the relay has enough antennas to perform zero-forcing decoding and precoding. Since we have $M_{1}^{1} \geq M_{2}^{1}, M_{1}^{2} \geq M_{2}^{2}$, we can let user 1 use only $M_{2}^{1}$ of its antennas and let user 3 use only $M_{2}^{2}$ of its antennas to transmit. The relay can decode all the messages and broadcast the messages to the intended users since it has sufficient spatial dimension.

Case 2: $N<2\left(M_{2}^{1}+M_{2}^{2}\right)$

For this case, we also have that $N \leq 2\left(M_{2}^{1}+M_{2}^{1}\right) \leq 2\left(M_{1}^{1}+\right.$ $\left.M_{2}^{1}\right)$. Depending on the number of antennas at the users and the relay, we need to further consider the following conditions:

Condition 1: $N \leq M_{1}^{1}$ and $N \leq M_{1}^{2}$. From Condition 2 in Lemma 1, the users in cluster 1 share $M_{2}^{1}$ dimensional signal space and the users in cluster 2 share $M_{2}^{2}$ dimensional space at the relay. Since we also have $N>M_{2}^{1}+M_{2}^{2}$, the users in each cluster can fully utilize their shared signal space at the relay to exchange messages. Specifically, users in cluster 1 and cluster 2 can transmit $M_{2}^{1}$ and $M_{2}^{2}$ data streams such that they are aligned at the relay, respectively. The relay decodes the sum of the messages and broadcast back to the intended clusters with proper receiver-side processing at the users. The DoF upper bound $2\left(M_{2}^{1}+M_{2}^{2}\right)$ can be achieved. The detailed scheme is similar to the previous cases and thus is omitted.

Condition 2: $M_{1}^{1}<N \leq M_{1}^{2}$. From Lemma 1, users in cluster 1 share $\left(M_{1}^{1}+M_{2}^{1}-N\right)^{+}$dimensional signal space, while the users in cluster 2 share $M_{2}^{2}$ dimensional signal space. Under this condition, we have $M_{1}^{1}+M_{2}^{1}-N<M_{2}^{1}$. Therefore using all the antennas at the relay and signal space alignment at two clusters cannot achieve the optimal DoF. In addition, since we have $N>M_{2}^{1}+M_{2}^{2}, M_{1}^{1}<N$ implies that $M_{1}^{1}+$ $M_{2}^{1}+M_{2}^{2}<2 N$, and thus the total dimension of shared signal space for the two clusters is less than $N$. Note that we do not have $M_{1}^{1}+M_{2}^{1}-N \geq 0$ for $N>M_{2}^{1}+M_{2}^{2}$, which is different from the case when $N \leq M_{2}^{1}+M_{2}^{2}$.

1) We first consider a scheme that allows the users in cluster 1 use the relay in the multiple-access fashion, and we also let the users in cluster 2 to use the shared $M_{2}^{2}$ dimensional space to perform signal space alignment. The dimension of signal space available for cluster 1 is $N-M_{2}^{2}$.
As long as $N-M_{2}^{2} \geq 2 M_{2}^{1}$, the users in cluster 1 can still exchange a total of $2 M_{2}^{1}$ messages using multiple-access type of schemes. The users in cluster 2 can always exchange $2 M_{2}^{2}$ messages using signal space alignment. Therefore, when $N \geq 2 M_{2}^{1}+M_{2}^{2}$, we can still achieve the DoF upper bound $2\left(M_{2}^{1}+M_{2}^{2}\right)$.

2) We next only allow the relay to use a subset of the antennas to assist the users. Specifically, if we have $M_{1}^{1} \geq$ $M_{2}^{1}+M_{2}^{2}$, we can let the relay use exactly $M_{2}^{1}+M_{2}^{2}$ antennas. From the result in Case 2 - Condition $1, N \leq M_{2}^{1}+M_{2}^{2}$, we can achieve DoF $2\left(M_{2}^{1}+M_{2}^{2}\right)$, which matches the upper bound.

3) When the conditions in the above cases are not satisfied, the scheme used achieves the DoF

$$
D o F=\max \left\{N+M_{2}^{2}, M_{1}^{1}+M_{2}^{1}+M_{2}^{2}\right\}
$$

Condition 3: $M_{1}^{2}<N \leq M_{1}^{1}$. The result for this case can be obtained following similar arguments from Case 2 Condition 2, $N>M_{2}^{1}+M_{2}^{2}$ and the details are omitted.

Condition 4: $N>M_{1}^{1}$ and $N>M_{1}^{2}$. Under this condition, we consider the following cases:

1) $M_{1}^{1} \geq M_{2}^{1}+M_{2}^{2}$ and $M_{1}^{2} \geq M_{2}^{1}+M_{2}^{2}$ : For this case, we have $N>M_{2}^{1}+M_{2}^{2}$. We can let the relay use only $M_{2}^{1}+$ $M_{2}^{2}$ antennas to assist the users, reducing this to Case 2 . Condition 1. $N \leq M_{2}^{1}+M_{2}^{2}$. The optimal DoF $2\left(M_{2}^{1}+M_{2}^{2}\right)$ can thus be achieved.

2) $M_{1}^{2} \geq 2 M_{2}^{1}+M_{2}^{2}$ : For this case, we have $N>2 M_{2}^{1}+M_{2}^{2}$. We can let the relay use only $2 M_{2}^{1}+M_{2}^{2}$ antennas to assist the users. Based on Lemma 1, the users in cluster 2 share $M_{2}^{2}$ dimensional signal space at the relay. We let the users in cluster 2 to use signal space alignment to exchange $2 M_{2}^{2}$ messages using $M_{2}^{2}$ dimensional space, while the users in cluster 1 use the rest $2 M_{2}^{1}$ dimensional space at the relay in the multiple-access fashion to exchange $2 M_{2}^{1}$ messages. The achieved DoF is thus $2\left(M_{2}^{1}+M_{2}^{2}\right)$, which matches the upper bound.

3) $M_{1}^{1} \geq M_{2}^{1}+2 M_{2}^{2}$ : For this case, the optimal DoF $2\left(M_{2}^{1}+\right.$ $M_{2}^{2}$ ) can be achieved following the same argument as in the previous case when $M_{1}^{2} \geq 2 M_{2}^{1}+M_{2}^{2}$.

4) For the other cases, we can always achieve the DoF $N$ by letting all the users transmit the data streams to the relay in the multiple-access fashion, and relay decodes all data streams and broadcasts back to the users. We can also let one cluster of users use signal space alignment, and the other cluster of users use multiple-access transmission. For example, we can let cluster 1 use $\left(M_{1}^{1}+M_{2}^{1}-N\right)^{+}$dimensional space to perform signal space alignment, and cluster 2 use the rest $N-\left(M_{1}^{1}+M_{2}^{1}-N\right)^{+}$dimensional space to perform multipleaccess transmission. The $\operatorname{DoF}\left(M_{1}^{1}+M_{2}^{1}-N\right)^{+}+N$ can thus be achieved. The $\operatorname{DoF}\left(M_{1}^{1}+M_{2}^{1}-N\right)^{+}+N$ can be achieved in a similar fashion. The last term in equation (51) can be achieved by using $\frac{M_{1}^{1}+M_{2}^{1}+M_{1}^{2}+M_{2}^{2}}{3}$ antennas at the relay, and the analysis is similar to Case 2 - Condition 4.(2), $N \leq M_{2}^{1}+M_{2}^{2}$ and the details are omitted.

Remark 9: Concurrent work [26] derives achievable schemes for multi-pair settings and these can also achieve the above optimal DoF for Condition 4, items 1), 2), 3) using all the antennas at the relay. 


\section{APPENDIX C}

\section{PROOF FOR THEOREM 3}

We first consider the DoF upperbound in Theorem 1. For the three user case, the upper bound reduces to

$$
\begin{aligned}
\text { DoF } \leq\{ & 2 N, M_{1}^{1}+M_{2}^{1}+M_{3}^{1}+M_{1}^{2}+M_{2}^{2}+M_{3}^{2}, \\
& 2\left(M_{2}^{1}+M_{3}^{1}+M_{2}^{2}+M_{3}^{2}\right), \\
& M_{1}^{1}+M_{2}^{1}+M_{3}^{1}+2\left(M_{2}^{2}+M_{3}^{2}\right), \\
& \left.2\left(M_{2}^{1}+M_{3}^{1}\right)+M_{1}^{2}+M_{2}^{2}+M_{3}^{2}\right\} .
\end{aligned}
$$

We now investigate the following cases to establish the optimal DoF:

1) When $2 N$ is the Binding Term in the DoF Upper Bound: For this case, we have

$$
\begin{aligned}
M_{1}^{1}+M_{2}^{1}+M_{3}^{1}+M_{1}^{2}+M_{2}^{2}+M_{3}^{2} & \geq 2 N, \\
M_{2}^{1}+M_{3}^{1}+M_{2}^{2}+M_{3}^{2} & \geq N .
\end{aligned}
$$

If we have $N \leq \max \left\{M_{2}^{1}, M_{2}^{2}\right\}$, the DoF $2 N$ can always be achieved by only letting the two users with more antennas than the relay to transmit using FDF schemes, which in fact reduces the channel to a two-way relay channel.

If we have $N>\max \left\{M_{2}^{1}, M_{2}^{2}\right\}$, we have $N>M_{2}^{1} \geq M_{3}^{1}$ and $N>M_{2}^{2} \geq M_{3}^{2}$. We consider the following scenarios:

- $M_{1}^{1} \geq N$ and $M_{1}^{2} \geq N$ : From Lemma 1 , the dimension of shared signal space between the users is summarized in Table VIII.

Since we have $M_{2}^{1}+M_{3}^{1}+M_{2}^{2}+M_{3}^{2} \geq N$, The DoF upper bound $2 N$ can thus be achieved by letting user 1 and user 2 , and user 1 and user 3 in each cluster to exchange messages using signal space alignment, such that all the dimension of the signal space of the relay is shared by one pair of users. The detailed scheme is similar to the two-user case and is thus omitted.

- $M_{1}^{1} \geq N$ and $N>M_{1}^{2}$ : From Lemma 1, the dimension of shared signal space between the users is summarized in Table IX.

If we have

$$
\begin{aligned}
& M_{2}^{1}+M_{3}^{1}+\left(M_{2}^{1}+M_{3}^{1}-N\right)^{+} \\
& \quad+\left(M_{1}^{2}+M_{2}^{2}-N\right)^{+}+\left(M_{1}^{2}+M_{3}^{2}-N\right)^{+} \\
& \quad+\left(M_{2}^{2}+M_{3}^{2}-N\right)^{+} \geq N,
\end{aligned}
$$

then all the dimension of the signal space at the relay can be shared by one pair of users. Using signal space alignment, user 1 and user 2 in cluster 1, user 1 and user 3 in cluster 1 , and the rest user pairs with $M_{i}^{l}+M_{k}^{l}-N>0$ can exchanges messages. The DoF upper bound $2 N$ can thus be achieved.

- $N>M_{1}^{1}$ and $M_{1}^{2} \geq N$ : From Lemma 1, the dimension of shared signal space between the users is summarized in Table X.

If we have

$$
\begin{aligned}
& \left(M_{1}^{1}+M_{2}^{1}-N\right)^{+}+\left(M_{1}^{1}+M_{3}^{1}-N\right)^{+} \\
& \quad+\left(M_{2}^{1}+M_{3}^{1}-N\right)^{+}+M_{2}^{2}+M_{3}^{2} \\
& \quad+\left(M_{2}^{2}+M_{3}^{2}-N\right)^{+} \geq N,
\end{aligned}
$$

then the DoF upper bound $2 N$ can be achieved following similar arguments as in the previous case.

- $N>M_{1}^{1}$ and $N>M_{1}^{2}$ : From Lemma 1 , the dimension of shared signal space between the users is summarized in Table XI.

If we have

$$
\begin{aligned}
\left(M_{1}^{1}\right. & \left.+M_{2}^{1}-N\right)^{+}+\left(M_{1}^{1}+M_{3}^{1}-N\right)^{+} \\
& +\left(M_{2}^{1}+M_{3}^{1}-N\right)^{+}+\left(M_{1}^{2}+M_{2}^{2}-N\right)^{+} \\
& +\left(M_{1}^{2}+M_{3}^{2}-N\right)^{+}+\left(M_{2}^{2}+M_{3}^{2}-N\right)^{+} \geq N,
\end{aligned}
$$

then the DoF upper bound $2 N$ can be achieved by letting user pairs with $M_{i}^{l}+M_{j}^{l}-N>0$ to exchange messages such that all dimension of the signal space of the relay is utilized by a pair of users using signal space alignment.

2) When $M_{1}^{1}+M_{2}^{1}+M_{3}^{1}+M_{1}^{2}+M_{2}^{2}+M_{3}^{2}$ is the Binding Term in the DoF Upper Bound: For this case, we have $M_{1}^{1} \leq M_{2}^{1}+M_{3}^{1}$ and $M_{1}^{2} \leq M_{2}^{2}+M_{3}^{2}$. The DoF upper bound $M_{1}^{1}+M_{2}^{1}+M_{3}^{1}+M_{1}^{2}+M_{2}^{2}+M_{3}^{2}$ can be achieved if $N \geq M_{1}^{1}+M_{2}^{1}+M_{3}^{1}+M_{1}^{2}+M_{2}^{2}+M_{3}^{2}$, i.e., users utilize the relay in the multiple-access fashion and relay can decode all the messages from the users and broadcast the messages to the intended users.

When $N<M_{1}^{1}+M_{2}^{1}+M_{3}^{1}+M_{1}^{2}+M_{2}^{2}+M_{3}^{2}$, it is easy to verify that the dimension of the shared signal space between all the users is always less than $\frac{M_{1}^{1}+M_{2}^{1}+M_{3}^{1}+M_{1}^{2}+M_{2}^{2}+M_{3}^{2}}{2}$, and whether the DoF upper bound can be achieved is unknown.

3) When $2\left(M_{2}^{1}+M_{3}^{1}+M_{2}^{2}+M_{3}^{2}\right)$ is the Binding Term in the DoF Upper Bound: For this case, we have $M_{1}^{1}>M_{2}^{1}+M_{3}^{1}$ and $M_{1}^{2}>M_{2}^{2}+M_{3}^{2}$. We also have $N>M_{2}^{1}+M_{3}^{1}+M_{2}^{2}+M_{3}^{2}$, which means user 2 and user 3 in each cluster cannot share any dimension of the signal space of the relay. The DoF upper bound can be achieved for the following cases:

- $N \geq 2\left(M_{2}^{1}+M_{3}^{1}+M_{2}^{2}+M_{3}^{2}\right)$ : Under this condition, the DoF upper bound can be achieved by letting all the users use the relay in the multiple-access fashion. The relay can decode all the messages and then broadcast the messages back to the intended users.

- $N<2\left(M_{2}^{1}+M_{3}^{1}+M_{2}^{2}+M_{3}^{2}\right)$ : Under this condition, the DoF upper bound can be achieve for the following cases:

- $M_{1}^{1} \geq M_{2}^{1}+M_{3}^{1}+M_{2}^{2}+M_{3}^{2}$ and $M_{1}^{2} \geq M_{2}^{1}+M_{3}^{1}+$ $M_{2}^{2}+M_{3}^{2}$ : The DoF upper bound can be achieved by only allowing the relay to use $M_{2}^{1}+M_{3}^{1}+M_{2}^{2}+M_{3}^{2}$ antennas to assist the users. Based on Lemma 1, the dimension of the shared signal space between the users is shown in Table XII. The DoF upper bound can thus be achieved by letting user 1 and user 2 , user 1 and user 3 in each cluster to exchange messages using signal space alignment.

- $N \geq 2\left(M_{2}^{1}+M_{3}^{1}\right)+M_{2}^{2}+M_{3}^{2}$ and $M_{1}^{2} \geq 2\left(M_{2}^{1}+\right.$ $\left.M_{3}^{1}\right)+M_{2}^{2}+M_{3}^{2}$ : The DoF upper bound can be achieved by only allowing the relay to use $2\left(M_{2}^{1}+\right.$ $\left.M_{3}^{1}\right)+M_{2}^{2}+M_{3}^{2}$ antennas to assist the users. Based on Lemma 1, user 1 and user 2 in cluster 2 share $M_{2}^{2}$ dimensional signal space, and user 1 and user 3 in cluster 2 share $M_{3}^{2}$ dimensional signal space. These pairs of users occupy $M_{2}^{2}+M_{3}^{2}$ dimensional signal 
TABLE VIII

\begin{tabular}{|l|l||l|l|}
\hline \multicolumn{2}{|c||}{ Cluster 1 } & \multicolumn{2}{c|}{ Cluster 2 } \\
\hline User 1 and 2 & $M_{2}^{1}$ & User 1 and 2 & $M_{2}^{2}$ \\
\hline User 1 and 3 & $M_{3}^{1}$ & User 1 and 3 & $M_{3}^{2}$ \\
\hline User 2 and 3 & $\left(M_{2}^{1}+M_{3}^{1}-N\right)^{+}$ & User 2 and 3 & $\left(M_{2}^{2}+M_{3}^{2}-N\right)^{+}$ \\
\hline
\end{tabular}

TABLE IX

\begin{tabular}{|l|l||l|l|}
\hline \multicolumn{2}{|c||}{ Cluster 1 } & \multicolumn{2}{c|}{ Cluster 2 } \\
\hline User 1 and 2 & $M_{2}^{1}$ & User 1 and 2 & $\left(M_{1}^{2}+M_{2}^{2}-N\right)^{+}$ \\
\hline User 1 and 3 & $M_{3}^{1}$ & User 1 and 3 & $\left(M_{1}^{2}+M_{3}^{2}-N\right)^{+}$ \\
\hline User 2 and 3 & $\left(M_{2}^{1}+M_{3}^{1}-N\right)^{+}$ & User 2 and 3 & $\left(M_{2}^{2}+M_{3}^{2}-N\right)^{+}$ \\
\hline
\end{tabular}

TABLE $\mathrm{X}$

\begin{tabular}{|l|l||l|l|}
\hline \multicolumn{2}{|c||}{ Cluster 1 } & \multicolumn{2}{c|}{ Cluster 2 } \\
\hline User 1 and 2 & $\left(M_{1}^{1}+M_{2}^{1}-N\right)^{+}$ & User 1 and 2 & $M_{2}^{2}$ \\
\hline User 1 and 3 & $\left(M_{1}^{1}+M_{3}^{1}-N\right)^{+}$ & User 1 and 3 & $M_{3}^{2}$ \\
\hline User 2 and 3 & $\left(M_{2}^{1}+M_{3}^{1}-N\right)^{+}$ & User 2 and 3 & $\left(M_{2}^{2}+M_{3}^{2}-N\right)^{+}$ \\
\hline
\end{tabular}

TABLE XI

\begin{tabular}{|l|l||l|l|}
\hline \multicolumn{2}{|c||}{ Cluster 1 } & \multicolumn{2}{c|}{ Cluster 2 } \\
\hline User 1 and 2 & $\left(M_{1}^{1}+M_{2}^{1}-N\right)^{+}$ & User 1 and 2 & $\left(M_{1}^{2}+M_{2}^{2}-N\right)^{+}$ \\
\hline User 1 and 3 & $\left(M_{1}^{1}+M_{3}^{1}-N\right)^{+}$ & User 1 and 3 & $\left(M_{1}^{2}+M_{3}^{2}-N\right)^{+}$ \\
\hline User 2 and 3 & $\left(M_{2}^{1}+M_{3}^{1}-N\right)^{+}$ & User 2 and 3 & $\left(M_{2}^{2}+M_{3}^{2}-N\right)^{+}$ \\
\hline
\end{tabular}

TABLE XII

\begin{tabular}{|l|l||l|l|}
\hline \multicolumn{2}{|c||}{ Cluster 1 } & \multicolumn{2}{c|}{ Cluster 2 } \\
\hline User 1 and 2 & $M_{2}^{1}$ & User 1 and 2 & $M_{2}^{2}$ \\
\hline User 1 and 3 & $M_{3}^{1}$ & User 1 and 3 & $M_{3}^{2}$ \\
\hline User 2 and 3 & 0 & User 2 and 3 & 0 \\
\hline
\end{tabular}

space at the relay, and can be used to exchange $2\left(M_{2}^{2}+M_{3}^{2}\right)$ messages. Users in cluster 1 can utilize the rest $2\left(M_{2}^{1}+M_{3}^{1}\right)$ dimensional signal space in the multiple-access fashion to exchange $2\left(M_{2}^{1}+M_{3}^{1}\right)$ messages. The DoF upper bound $2\left(M_{2}^{1}+M_{3}^{1}+M_{2}^{2}+\right.$ $\left.M_{3}^{2}\right)$ can thus be achieved.

- $N \geq M_{2}^{1}+M_{3}^{1}+2\left(M_{2}^{2}+M_{3}^{2}\right)$ and $M_{1}^{1} \geq M_{2}^{1}+$ $M_{3}^{1}+2\left(M_{2}^{2}+M_{3}^{2}\right)$ : The DoF upper bound $2\left(M_{2}^{1}+\right.$ $\left.M_{3}^{1}+M_{2}^{2}+M_{3}^{2}\right)$ can be achieved by only allowing the relay to use $M_{2}^{1}+M_{3}^{1}+2\left(M_{2}^{2}+M_{3}^{2}\right)$ antennas, following similar arguments as in the previous case.

4) When $M_{1}^{1}+M_{2}^{1}+M_{3}^{1}+2\left(M_{2}^{2}+M_{3}^{2}\right)$ is the Binding Term in the DoF Upper Bound: For this case we have $M_{1}^{1}<M_{2}^{1}+M_{3}^{1}$ and $M_{1}^{2}>M_{2}^{2}+M_{3}^{2}$. We also have

$$
N>\frac{M_{1}^{1}+M_{2}^{1}+M_{3}^{1}}{2}+M_{2}^{2}+M_{3}^{2} .
$$

The DoF upper bound can be achieved for the following cases:

- $N \geq M_{1}^{1}+M_{2}^{1}+M_{3}^{1}+2\left(M_{2}^{2}+M_{3}^{2}\right)$ : The DoF upper bound can be simply achieved by letting the users exchange their messages using the relay in the multiple-access fashion. The relay can decode all the messages and broadcast the messages back to the intended users since it has sufficient spatial dimension.

- $M_{1}^{1}+M_{2}^{1}+M_{3}^{1}+M_{2}^{2}+M_{3}^{2} \leq N<M_{1}^{1}+M_{2}^{1}+M_{3}^{1}+$ $2\left(M_{2}^{2}+M_{3}^{2}\right)$ and $M_{1}^{2} \geq M_{1}^{1}+M_{2}^{1}+M_{3}^{1}+M_{2}^{2}+M_{3}^{2}$ : The DoF upper bound can be achieved by only allowing the relay to use $M_{1}^{1}+M_{2}^{1}+M_{3}^{1}+M_{2}^{2}+M_{3}^{2}$ antennas to assist the users. Based on Lemma 1, user 1 and user 2 in cluster 2 share $M_{2}^{2}$ dimensional space, and user 1 and user 3 in cluster 2 share $M_{3}^{2}$ dimensional space at the relay, which allows the users to exchange $2\left(M_{2}^{2}+M_{3}^{2}\right)$ messages using $M_{2}^{2}+M_{3}^{2}$ dimensional space at the relay. The rest $M_{1}^{1}+M_{2}^{1}+M_{3}^{1}$ dimensional signal space at the relay can be used to assist users in cluster 1 to exchange $M_{1}^{1}+M_{2}^{1}+M_{3}^{1}$ messages in the multiple-access fashion. Therefore the DoF $M_{1}^{1}+M_{2}^{1}+M_{3}^{1}+2\left(M_{2}^{2}+M_{3}^{2}\right)$ can be achieved.

5) When $2\left(M_{2}^{1}+M_{3}^{1}\right)+M_{1}^{2}+M_{2}^{2}+M_{3}^{2}$ is the Binding Term in the DoF Upper Bound: For this case, the DoF upper bound can be achieved for the following scenarios:

- $N \geq 2\left(M_{2}^{1}+M_{3}^{1}\right)+M_{1}^{2}+M_{2}^{2}+M_{3}^{2}$.

- $M_{2}^{1}+M_{3}^{1}+M_{1}^{2}+M_{2}^{2}+M_{3}^{2} \leq N<2\left(M_{2}^{1}+M_{3}^{1}\right)+M_{1}^{2}+$ $M_{2}^{2}+M_{3}^{2}$ and $M_{1}^{1} \geq M_{2}^{1}+M_{3}^{1}+M_{1}^{2}+M_{2}^{2}+M_{3}^{2}$.

This case is similar to case 4 ), and the details are thus omitted. 


\section{REFERENCES}

[1] Y. Tian and A. Yener, "Signal space alignment and degrees of freedom for the two-cluster multi-way relay channel," in Proc. IEEE Int. Conf. Commun. China, Aug. 2012, pp. 12-17.

[2] Y. Tian and A. Yener, "Degrees of freedom optimal transmission for the two-cluster MIMO multi-way relay channel," in Proc. IEEE Int. Conf. Commun., Jun. 2013, pp. 3344-3348.

[3] Y. Tian and A. Yener, "Degrees of freedom for the MIMO multiway relay channel," in Proc. IEEE Int. Symp. Inf. Theory, Jul. 2013, pp. 1576-1580.

[4] D. Gündüz, A. Yener, A. J. Goldsmith, and H. V. Poor, "The multiway relay channel," IEEE Trans. Inf. Theory, vol. 59, no. 1, pp. 51-63, Jan. 2013.

[5] C. E. Shannon, "Two-way communication channels," in Proc. 4th Berkeley Symp. Math, Statist. Probab., 1961, pp. 611-644.

[6] A. S. Avestimehr, A. Sezgin, and D. Tse, "Capacity of the two-way relay channel within a constant gap," Eur. Trans. Telecommun., vol. 21, no. 4, pp. 363-374, 2009.

[7] W. Nam, S. Y. Chung, and Y. H. Lee, "Capacity of the Gaussian twoway relay channel to within $1 / 2$ bit," IEEE Trans. Inf. Theory, vol. 56, no. 11, pp. 5488-5494, Nov. 2011.

[8] M. P. Wilson, K. Narayanan, H. Pfister, and A. Sprintson, "Joint physical layer coding and network coding for bi-directional relaying," IEEE Trans. Inf. Theory, vol. 56, no. 11, pp. 5641-5654, Nov. 2010.

[9] M. Chen and A. Yener, "Power allocation for F/TDMA multiuser twoway relay networks," IEEE Trans. Wireless Commun., vol. 9, no. 2, pp. 546-551, Feb. 2010.

[10] L. Ong, S. J. Johnson, and C. M. Kellett, "The capacity region of multiway relay channels over finite fields with full data exchange," IEEE Trans. Inf. Theory, vol. 57, no. 5, pp. 3016-3031, May 2011.

[11] L. Ong, C. M. Kellett, and S. J. Johnson, "On the equal-rate capacity of the AWGN multiway relay channel," IEEE Trans. Inf. Theory, vol. 58, no. 9, pp. 5761-5769, Sep. 2012.

[12] A. Chaaban and A. Sezgin, "The capacity region of the linear shift deterministic Y-channel," in Proc. IEEE Int. Symp. Inf. Theory, Jul. 2011, pp. 2457-2461.

[13] A. Chaaban, A. Sezgin, and A. S. Avestimehr, "On the sum capacity of the Y-channel," in Proc. 45th Asilomar Conf. Signals, Syst. Comput., Nov. 2011, pp. 2135-2139.

[14] A. Sezgin, A. S. Avestimehr, M. A. Khajehnejad, and B. Hassibi, "Divide-and-conquer: Approaching the capacity of the two-pair bidirectional Gaussian relay network," IEEE Trans. Inf. Theory, vol. 58, no. 4, pp. 2434-2454, Apr. 2012

[15] M. Chen and A. Yener, "Multiuser two-way relaying: Detection and interference management strategies," IEEE Trans. Wireless Commun., vol. 8, no. 8, pp. 4296-4305, Aug. 2009.

[16] V. R. Cadambe and S. A. Jafar, "Interference alignment and the degrees of freedom for the K-user interference channel," IEEE Trans. Inf. Theory, vol. 54 , no. 8, pp. 3425-3441, Aug. 2008.

[17] S. A. Jafar and S. Shamai, "Degrees of freedom region for the MIMO X channel," IEEE Trans. Inf. Theory, vol. 54, no. 1, pp. 151-170, Jan. 2008

[18] V. R. Cadambe and S. A. Jafar, "Interference alignment and the degrees of freedom of wireless X networks," IEEE Trans. Inf. Theory, vol. 55, no. 9, pp. 3893-3908, Sep. 2009.

[19] T. Gou and S. A. Jafar, "Degrees of freedom of the K user MxN MIMO interference channel," IEEE Trans. Inf. Theory, vol. 56, no. 12 pp. 6040-6057, Dec. 2010.

[20] N. Lee, J.-B. Lim, and J. Chun, "Degrees of freedom of the MIMO Y channel: Signal space alignment for network coding," IEEE Trans. Inf. Theory, vol. 56, no. 7, pp. 3332-3342, Jul. 2010.

[21] K. Lee, N. Lee, and I. Lee, "Achievable degrees of freedom on K-user Y channels," IEEE Trans. Wireless Commun., vol. 11, no. 3, pp. 1210-1219, Mar. 2012.
[22] R. S. Ganesan, T. Weber, and A. Klein, "Interference alignment in multi-user two way relay networks," in Proc. IEEE 73rd Veh. Technol. Conf., May 2011, pp. 1-5.

[23] K. Lee, S.-H. Park, J.-S. Kim, and I. Lee, "Degrees of freedom on MIMO multi-link two-way relay channels," in Proc. IEEE Global Telecommun. Conf., Dec. 2010, pp. 1-5.

[24] F. Sun and E. de Carvalho, "Degrees of freedom of asymmetrical multi-way relay network," in Proc. IEEE 12th Int. Workshop Signal Process. Adv. Wireless Commun., Jun. 2011, pp. 531-535.

[25] U. Erez and R. Zamir, "Achieving $1 / 2 \log (1+S N R)$ on the AWGN channel with lattice encoding and decoding," IEEE Trans. Inf. Theory, vol. 50, no. 10, pp. 2293-2314, Oct. 2004.

[26] R. S. Ganesan, H. Al-Shatri, A. Kuehne, T. Weber, and A. Klein, "Pair-aware interference alignment in multi-user two-way relay networks," IEEE Trans. Wireless Commun., vol. 12, no. 8, pp. 3662-3671, Aug. 2013.

Ye Tian (S'10) received the B.E. degree in information engineering from Beijing University of Posts and Telecommunications, Beijing, China, in 2007. Between 2007 and 2013 he was a research assistant at the Wireless Communications and Networking Laboratory at the Pennsylvania State University (WCAN@PSU). He received the Ph.D. degree in Electrical Engineering from the Pennsylvania State University, University Park, PA, in 2013. His Ph.D. dissertation received the Dr. Nirmal K. Bose Dissertation Excellence Award, and his doctoral research received the Melvin P. Bloom Memorial Outstanding Doctoral Research Award, both from Penn State. Dr. Tian's research interests include network information theory with particular emphasis on impact of cooperation and interference in wireless networks. He was a student co-chair of the IEEE Information Theory Society Student Committee in 2010-2012. Currently, he is a scientist of system design at Broadcom, Sunnyvale, CA.

Aylin Yener (S'91-M'00-SM'13) received the B.Sc. degree in electrical and electronics engineering, and the B.Sc. degree in physics, from Boğaziçi University, Istanbul, Turkey; and the M.S. and Ph.D. degrees in electrical and computer engineering from Wireless Information Network Laboratory (WINLAB), Rutgers University, New Brunswick, NJ. Commencing fall 2000 , for three semesters, she was a P.C. Rossin Assistant Professor at the Electrical Engineering and Computer Science Department, Lehigh University, PA. In 2002, she joined the faculty of The Pennsylvania State University, University Park, PA, where she was an Assistant Professor, then Associate Professor, and is currently Professor of Electrical Engineering since 2010. During the academic year 2008-2009, she was a Visiting Associate Professor with the Department of Electrical Engineering, Stanford University, CA. Her research interests are in information theory, communication theory and network science, with recent emphasis on green communications and information security. She received the NSF CAREER award in 2003.

Dr. Yener previously served as a technical program chair or co-chair for various conferences for the IEEE Communications Society, as an associate editor for the IEEE TRANSACTIONS ON COMMUNICATIONS, as an associate editor and an editorial advisory board member for the IEEE TRANS ACTIONS ON WIRELESS COMMUNICATIONS. She served as the student committee chair for the IEEE Information Theory Society 2007-2011, and was the co-founder of the Annual School of Information Theory in North America co-organizing the school in 2008, 2009 and 2010. Dr. Yener currently serves on the board of governors of the IEEE Information Theory Society as its treasurer. 